\title{
Immune exclusion by naturally acquired secretory IgA against pneumococcal pilus-1
}

\author{
Ulrike Binsker, John A. Lees, Alexandria J. Hammond, and Jeffrey N. Weiser \\ Department of Microbiology, New York University School of Medicine, New York, New York, USA.
}

\begin{abstract}
Successful infection by mucosal pathogens requires overcoming the mucus barrier. To better understand this key step, we performed a survey of the interactions between human respiratory mucus and the human pathogen Streptococcus pneumoniae. Pneumococcal adherence to adult human nasal fluid was seen only by isolates expressing pilus-1. Robust binding was independent of pilus-1 adhesive properties but required Fab-dependent recognition of RrgB, the pilus shaft protein, by naturally acquired secretory IgA (sIgA). Pilus-1 binding by specific slgA led to bacterial agglutination, but adherence required interaction of agglutinated pneumococci and entrapment in mucus particles. To test the effect of these interactions in vivo, pneumococci were preincubated with human slgA before intranasal challenge in a mouse model of colonization. slgA treatment resulted in rapid immune exclusion of pilus-expressing pneumococci. Our findings predict that immune exclusion would select for nonpiliated isolates in individuals who acquired RrgB-specific slgA from prior episodes of colonization with piliated strains. Accordingly, genomic data comparing isolates carried by mothers and their children showed that mothers are less likely to be colonized with pilus-expressing strains. Our study provides a specific example of immune exclusion involving naturally acquired antibody in the human host, a major factor driving pneumococcal adaptation.
\end{abstract}

\section{Introduction}

Airway mucus plays a critical role in host defense by providing a physicochemical barrier that protects the underlying respiratory epithelium from inhaled particulate matter, including infectious agents $(1,2)$. The mucus forms a semipermeable network, which allows the transport of water, nutrients, and gases, and facilitates mucosal defense involving innate and adaptive immunity (3). Moreover, the adhesive properties of mucus trap bacteria as well as other particles, promoting their mechanical clearance through mucociliary movements (1). The mucus is divided into 2 layers: an inner periciliary layer, which is relatively impenetrable to microbes, and an outer loose layer providing a niche for commensal bacteria that is continuously removed by ciliary activity (4). The nasal mucus is composed of $95 \%$ water, high-molecular weight glycoproteins (so called mucins, which confer the viscous properties of the mucus), lipids, proteins, and inorganic salts (5). Nearly all of the proteinaceous components present in the mucus harbor antibacterial activity (6-8). Given the importance of the mucus layer in maintaining airway homeostasis, there is little mechanistic understanding of bacterial-mucus interactions.

Streptococcus pneumoniae (Spn, the pneumococcus) is an opportunistic pathogen of the human upper respiratory tract with colonization rates of $25 \%-65 \%$ in children and $5 \%-10 \%$ in the adult population $(9,10)$. Carriage is usually asymptomatic; however, under certain circumstances the pneumococcus gains access to normally sterile sites, leading to invasive infections such as

Conflict of interest: The authors have declared that no conflict of interest exists. Copyright: () 2020, American Society for Clinical Investigation.

Submitted: July 22, 2019; Accepted: October 30, 2019; Published: January 13, 2020

Reference information: / Clin Invest. 2020;130(2):927-941.

https://doi.org/10.1172/JCl132005. otitis media, pneumonia, sepsis, and meningitis (11). Successful colonization requires penetration through the mucus barrier protecting the respiratory epithelium. Spn has evolved several strategies to overcome the mucus layer and to evade mucociliary clearance. Most strains are surrounded by a thick capsule composed of negatively charged polysaccharide that repels anionic mucins and other mucus glycoproteins (12). The amount of capsule and its serotype affect binding to mucus, which is inversely correlated with persistence during early colonization. Capsule-dependent release from mucus entrapment also allows for bacterial shedding and host-to-host transmission following contact with nasal secretions (13). Furthermore, Spn expresses multiple exo- and endoglycosidases able to degrade $\mathrm{O}-$ and $\mathrm{N}$-linked glycans of mucosal proteins (14-16). Mucus components, including lactoferrin, secretory component, secretory immunoglobulin A (sIgA), and mucins, have been shown to be substrates of Spn glycosidases (14, $17,18)$. Potential changes in the integrity and protective function of mucus by Spn glycosidases might contribute to the movement of the bacterium through the mucus layer. Additionally, cleaved carbohydrates serve as a carbon source in the normally nutrient-poor environment of the nasopharynx (19). Spn also alters the mucus composition via its major toxin pneumolysin, which triggers the upregulation of Muc5AC, a prominent secretory mucin in the airways (20). This excessive mucus production could overwhelm the effectiveness of mucociliary flow and increase nasal discharge, allowing for pneumococcal transmission (21).

Herein, we evaluated the interactions of Spn with respiratory mucus. We identified bacterial components and mucus factors involved in binding of Spn and impacting colonization. Since Spn is a human-specific organism, we focused on its interaction with human nasal secretions. We found that the pneumococcal pilus-1 is the major determinant of Spn binding to human mucus. Fur- 

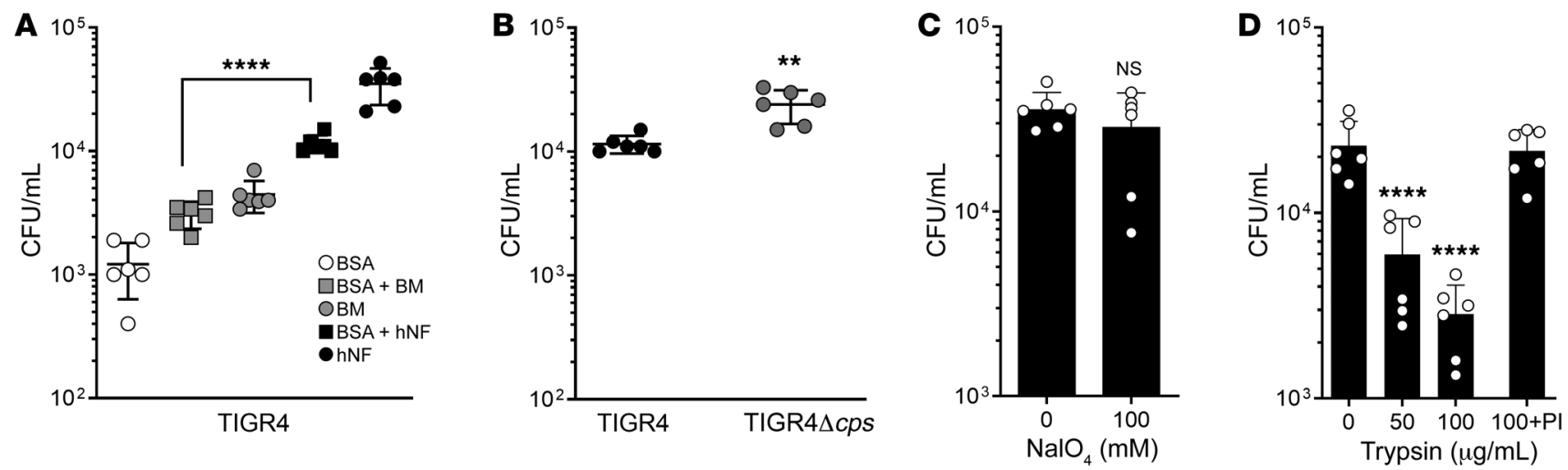

Figure 1. Mucosal protein-mediated binding of Spn to human nasal fluid. (A-D) Adherence of Spn TIGR4 to human nasal fluid (hNF) was analyzed in a solid-phase assay. (A) Bacteria $\left(1 \times 10^{4}\right)$ in $100 \mu \mathrm{L}$ DMEM were incubated with $10 \mu \mathrm{g}$ immobilized bovine submaxillary mucus (BM) or hNF in the presence or absence of $0.1 \% \mathrm{BSA}$ for 2 hours at $30^{\circ} \mathrm{C}$. Bound bacteria were determined by resuspension with $0.001 \%$ Triton X-100 following plating on TS agar plates supplemented with $200 \mu \mathrm{g} / \mathrm{mL}$ streptomycin. (B) Adherence of TIGR4 and TIGR4 $\Delta$ cps (each $1 \times 10^{4}$ per $100 \mu \mathrm{L}$ ) to hNF. (C) Treatment of immobilized hNF with $100 \mathrm{mM} \mathrm{NaIO}_{4}$ in $50 \mathrm{mM}$ sodium acetate buffer for 30 minutes at $4^{\circ} \mathrm{C}$ in the dark followed by blocking with $0.1 \%$ BSA and incubation with $2 \times 10^{4}$ Spn TIGR4. (D) Immobilized hNF was incubated with increasing concentrations of trypsin with or without protease inhibitor (PI) for 30 minutes at $37^{\circ} \mathrm{C}$ followed by incubation of $0.1 \%$ BSA and $2 \times 10^{4} \mathrm{Spn}$ TICR4 in $100 \mu \mathrm{L}$ DMEM for 2 hours at $30^{\circ} \mathrm{C}$. Experiments were performed in duplicate, and mean values of 3 independent experiments are shown with error bars corresponding to SD. ${ }^{* *} P<0.01,{ }^{* * *} P<0.0001$ by 2-tailed unpaired $t$ test, $n=6$ (A, B, and $\mathbf{D}$ ), or 1-way ANOVA followed by Dunnett's multiple comparison, $n=6$ (C).

thermore, we show that naturally acquired sIgA mediates pilusdependent agglutination, facilitating binding to mucus, and that this interaction inhibits the establishment of colonization in a murine model. Our study provides mechanistic insight into the interactions of Spn with mucus and may explain the low abundance of pilus-1 among clinical pneumococcal isolates, particularly after childhood exposure when pilus-specific sIgA has accumulated. Furthermore, we provide a demonstration of host defense mediated by mucosal antigen-specific sIgA (referred to as immune exclusion) $(22,23)$.

\section{Results}

Pneumococci interact with human nasal mucus via mucosal proteins. Colonizing Spn are found predominantly within the glycocalyx, the mucus layer overlaying the epithelial surface (12). We established an in vitro assay to study Spn interactions with human mucus, considering both attachment and detachment. The association of encapsulated Spn (isolate TIGR4) with immobilized pooled human nasal fluid (hNF) collected from healthy adults was quantified using a solid-phase assay with BSA as blocking reagent. Spn adhered to hNF more readily compared with bovine submaxillary mucus, which has been recently used in a similar approach (13) (Figure 1A). Adherence to either source of mucus was higher than in controls with BSA alone. As a control for the functionality of the assay, we demonstrate that adherence of an isogenic capsuledeficient mutant to hNF was significantly increased as previously described for bovine submaxillary mucus (Figure 1B) (13).

Next, we considered whether Spn interactions with hNF involved binding to host carbohydrate moieties and/or protein(s). hNF was pretreated with either sodium periodate $\left(\mathrm{NaIO}_{4}\right)$ to oxidize oligosaccharides or trypsin to degrade protein. Even high concentrations of sodium periodate did not affect the binding of Spn to hNF (Figure 1C). In contrast, incubation of hNF with increasing concentrations of trypsin reduced the adherence of Spn in a concentration-dependent manner (Figure 1D). Inhibition of trypsin activity restored adherence levels equivalent to those without added protease.
Three of the most abundant proteins in hNF are lactoferrin, sIgA, and lysozyme (Figure 2, A-C, and ref. 24). Pneumococcal surface protein A (PspA) and pilus-1 have been shown to bind to purified human lactoferrin $(25,26)$. In addition, pneumococcal surface protein $\mathrm{C}(\mathrm{PspC} / \mathrm{CbpA})$ interacts specifically with the secretory component of human $\operatorname{sIgA}(27,28)$. Furthermore, a recent study suggests the interaction of pilus-1 with the extracellular domain of polymeric immunoglobulin receptor (pIgR) that is identical to secretory component (29). To assess the relevance of these interactions in human nasal secretions, we incubated whole TIGR4 or defined mutants lacking these surface proteins with soluble hNF and detected bound lactoferrin or sIgA by flow cytometry. Our data confirmed that PspA is the major protein responsible for lactoferrin recruitment to the Spn surface (Figure 2D, and dot plots in Supplemental Figure 1; supplemental material available online with this article; https://doi.org/10.1172/JCI132005DS1). The mutant lacking the entire pathogenicity islet expressing pilus-1 did not display impaired binding of mucosal lactoferrin. However, lack of pilus- 1 resulted in a $72 \%$ reduction in binding of sIgA in comparison with the isogenic parental strain. The $p s p C$-deletion mutant also showed a significantly impaired binding of mucosal sIgA, though to a lesser extent than the pilus-deficient mutant. Loss of PspA did not alter the acquisition of sIgA to the bacterial surface. PspA, PspC, and pilus-1 are immunogenic in humans, and antibodies against these surface factors, which likely result from prior exposure during colonization episodes, are common (30-34). Therefore, we also analyzed the binding of IgG in hNF to Spn and found no significant difference between WT Spn and the protein-deficient mutants. Overall, the results demonstrate a role for interactions between bacterial factors and host mucosal proteins: PspA-lactoferrin and pilus-1/PspC-sIgA.

Pilus-1 is the major factor interacting with human mucus. A minority of clinical Spn isolates express pilus-1 (35-37). We screened clinical isolates for pilus expression by immunoblotting and confirmed the presence or absence of the pilus- 1 pathogenicity islet by PCR 
A
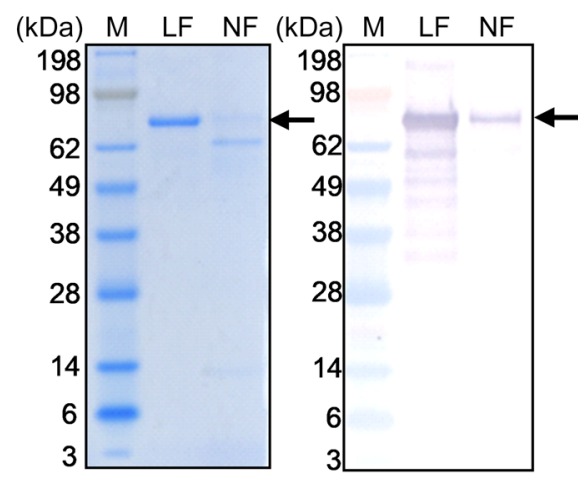

D

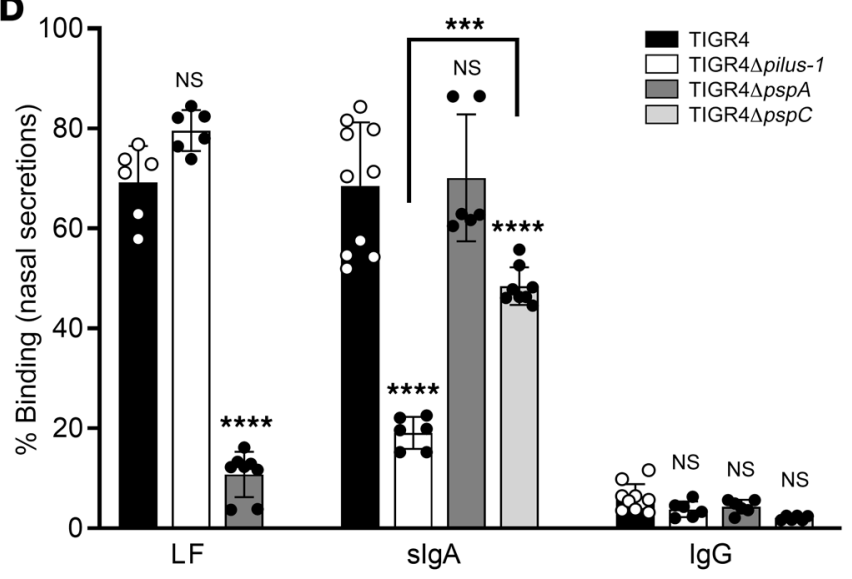

B

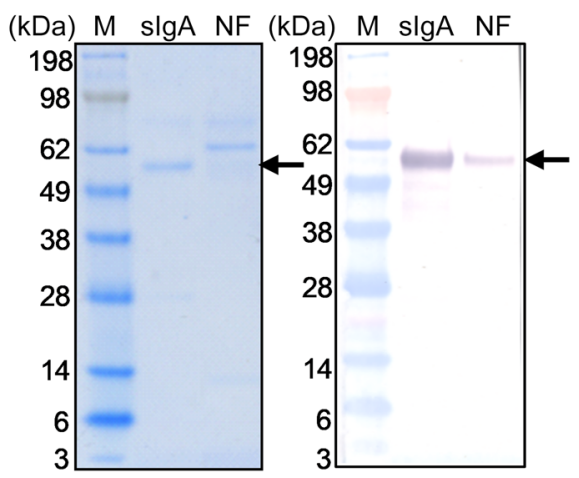

C

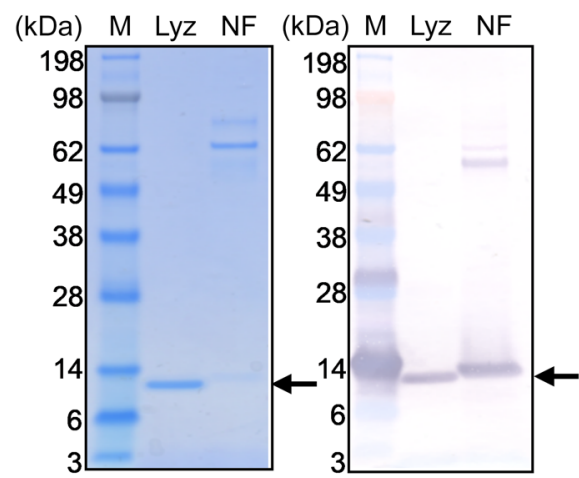

\begin{abstract}
Figure 2. Major pneumococcal surface proteins interacting with human mucus proteins. (A-C) Gradient SDS-PAGE (4\%-12\%) and Western blot of purified mucosal proteins lactoferrin (LF), slgA, lysozyme (Lyz), and hNF (each $1 \mu \mathrm{g}$ ). Proteins in hNF were detected using a specific polyclonal rabbit anti-human lactoferrin antibody (A), an alkaline phosphatase-coupled goat anti-human IgA antibody (B), or a rabbit anti-human lysozyme antibody (C). Protein band at $62 \mathrm{kDa}$ likely represents albumin. Lane M, SeeBlue Plus2 Pre-Stained Standard (Thermo Fisher Scientific). (D) Acquisition of soluble LF, slgA, and IgC from hNF by Spn. Bacteria $\left(5 \times 10^{6} \mathrm{CFU}\right.$ per $50 \mu \mathrm{L}$ ) were incubated with $50 \mu \mathrm{g} / \mathrm{mL}$ of hNF. Binding of bacteria-associated LF, slgA, and IgC was analyzed by flow cytometry. The percentage binding of at least 3 independent experiments is shown as mean values with error bars \pm SD. ${ }^{* *} P<0.001,{ }^{* * *} P<0.0001$ by 1 -way ANOVA followed by Tukey's multiple comparison, $n=6-10$.
\end{abstract}

using primers within $\operatorname{rrgB}$ (Supplemental Figure 2). To examine a contribution of pilus-1 in Spn adherence to hNF, we used, in addition to the TIGR4 strain, 2 piliated (types $9 \mathrm{~V}$ and 19F) and 2 nonpiliated (types $6 \mathrm{~A}$ and $23 \mathrm{~F}$ ) clinical isolates. Over a time period of 5 hours, adherence levels of the pilus-expressing strains were similar to those of the TIGR4 reference strain, while adherence of the nonpiliated strains was significantly lower in comparison with TIGR4 (Figure 3A). Detachment was quantified as a further measure of the strength of interaction between Spn and hNF. In comparison with piliated strains, the nonpiliated strains detached significantly more readily from the nasal mucus (Figure $3 \mathrm{~B}$ ). To further confirm the role of pilus- 1 in Spn binding to immobilized hNF, we used pilus-1-deficient constructs of the TIGR4, 9V, and 19F strains and a pilus-1 islet-knockin mutant of the $23 \mathrm{~F}$ strain. Again, adherence to hNF correlated with pilus expression over genetic background (Figure $3, \mathrm{C}-\mathrm{F}$ ). In addition, the $\operatorname{rrg} A$ and $\operatorname{rrg} B$ genes of the $9 \mathrm{~V}$ and $19 \mathrm{~F}$ strain were sequenced, and both belong to the common clades that include TIGR4 (Supplemental Figures 3 and 4). Overall, adherence to hNF of the mutants without the pilus-1 islet was reduced 4- to 14 -fold. Furthermore, adherence of the piliated strain was reduced to the levels of the nonpiliated mutant by pretreatment of hNF with trypsin (Supplemental Figure 5). In contrast to pilus-1, we were unable to detect a role for PspA or PspC in adherence to immobilized hNF (Figure 3, C and F) despite the role of these Spn proteins in binding factors in human nasal secretions (Figure 2D, and dot plots in Supplemental Figure 1). Our findings show that pilus-1 is the major Spn surface structure mediating binding of Spn to normal human nasal mucus from adults.
Next, we determined which specific component of the pilus is involved in binding to human mucus. Pilus-1 is composed of 3 subunits: the tip RrgA, which is also described as adhesin; the shaft RrgB; and the ancillary protein $\operatorname{RrgC}(26,37-40)$. We performed solid-phase assays and flow cytometry using pneumococcal mutants, which lacked 1 or 2 pilus- 1 components. Adherence of the $r r g A$-deficient strain was slightly but significantly reduced in comparison with WT (Figure 4A). The $\operatorname{rrgB}$-deletion mutant was most impaired in adherence to hNF. The absence of the pilus-1 subunit RrgC did not impact Spn adherence, and loss of both RrgB and $\mathrm{RrgC}$ resulted in levels comparable to those of the single $\mathrm{rrgB}$ deletion mutant (Figure 4A). Additionally, recruitment of sIgA from soluble hNF by each of the mutants directly correlated with their ability to adhere to immobilized mucus (Figure 4B, and dot plots in Supplemental Figure 6). To further verify a contribution of RrgA and RrgB in binding to hNF, we performed an inhibition assay using specific antibodies against these pilus-1 components. WT Spn pretreated with either anti-RrgA or anti-RrgB antisera adhered significantly less to hNF compared with controls (Figure 4C). Blocking of $\mathrm{RrgB}$ resulted in a greater inhibitory effect and consequently lower levels of Spn adherence compared with blocking of RrgA. As expected, the pretreatment of the pilus-1-deficient mutant with the RrgA- and RrgB-specific antibodies had no effect on adherence to hNF (Figure 4C). Together, these findings suggest that the pilus-1 shaft-forming subunit RrgB is the main Spn factor bound by human mucus, with a small contribution from the tip component RrgA.

Pneumococcal pilus-1 is a major determinant responsible for human sIgA recruitment. Based on the impaired sIgA binding of the 

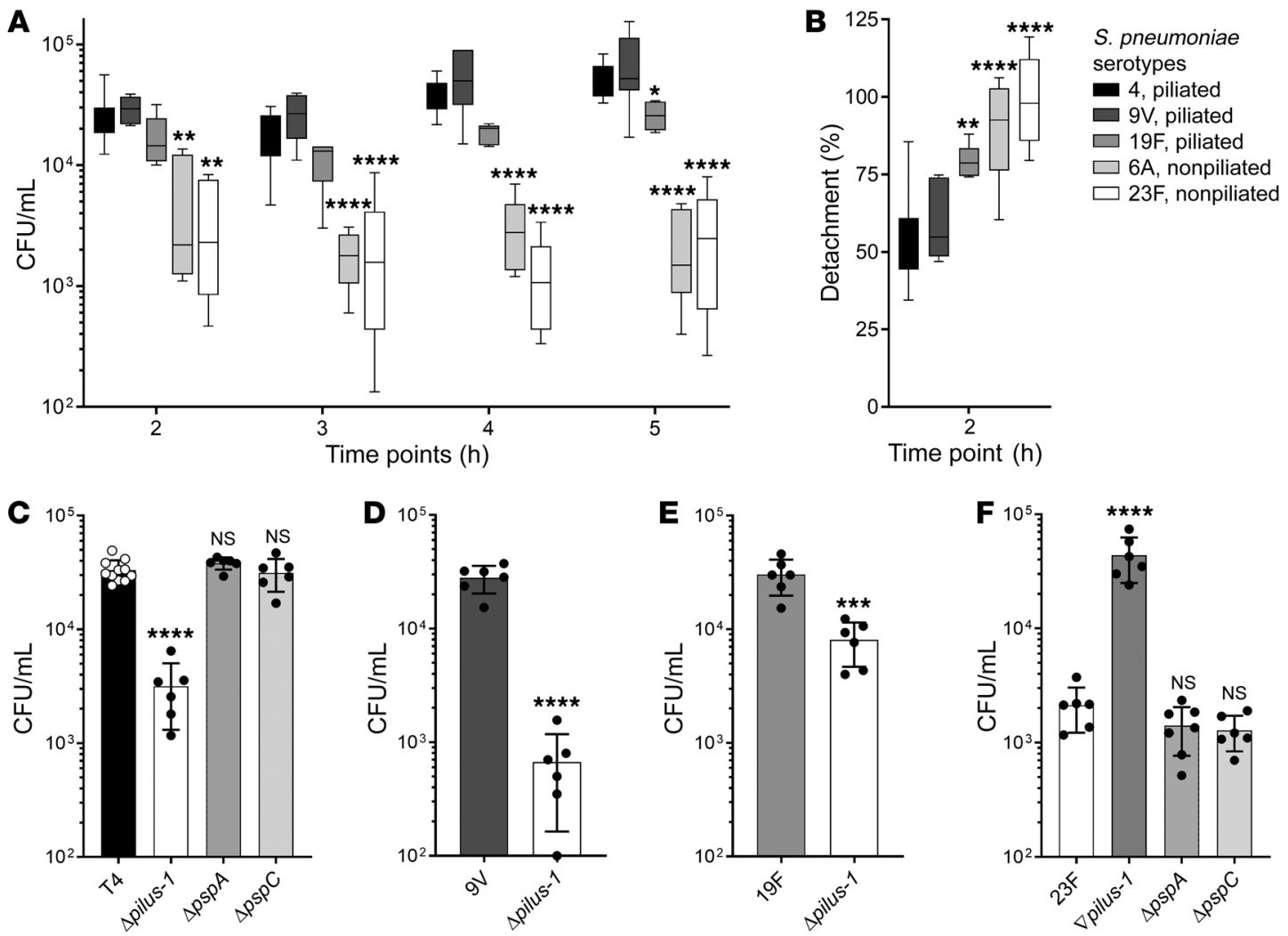

Figure 3. Pilus-1-mediated adherence of Spn to hNF. (A and B) Adherence to and detachment from hNF using pilus-1-expressing (T4 [TIGR4], 9V, 19F) and nonpiliated (6A, 23F) clinical isolates. (A) Adherence of Spn $\left(2 \times 10^{4}\right.$ per $\left.100 \mu \mathrm{L}\right)$ was observed over a time period of 5 hours; $n=6-12$. (B) Detachment (unbound bacteria recovered after 19 washing steps) is calculated as the percentage of the total CFU per well after 2 hours of incubation; $n=6-12$. (C-F) Adherence of WT Spn and isogenic mutants after 2 hours of incubation with immobilized hNF; $n=6-11$. Experiments were performed in duplicate, and values of at least 3 independent experiments are shown in box-and-whiskers columns including minimal and maximal value ( $\mathbf{A}$ and $\mathbf{B})$ or as bar graphs $\pm \mathrm{SD}$. ${ }^{*} P<0.05,{ }^{* *} P<0.01,{ }^{* *} P<0.001,{ }^{* * * *} P<0.0001$ by 1 -way ANOVA followed by Šidák's multiple comparison vs. T4 (A) or by Dunnett's multiple comparison (B, C, and $\mathbf{F}$ ) or 2-tailed unpaired $t$ test ( $\mathbf{D}$ and $\mathbf{E}$ ) vs. T4 or WT.

pilus-1-deficient mutant when incubated with hNF, we analyzed the acquisition of human SIgA (purified from pooled colostrum) to the Spn surface using flow cytometry. Because of the binding of PspC to the human secretory component of $\operatorname{sIgA}$, a $p s p C$-deficient mutant was used as control. Secretory IgA bound in a concentration-dependent fashion to the surface of the TIGR4 as well as to the isogenic pilus-1- and pspC-deficient mutants (Figure 5A). Deletion of the pilus-1 islet decreased sIgA acquisition by up to $78 \%$, whereas the lack of PspC reduced binding of sIgA only up to $38 \%$. To decipher whether binding of this immunoglobulin isotype is exclusive for sIgA, we also analyzed binding of pooled human serum IgA. Based on the dimeric structure of sIgA and the resulting tetrameric valence of the molecule, monomeric divalent serum IgA was used in a 2 -fold molar ratio. Interestingly, recruitment of serum IgA by the nonpiliated mutant was lower in comparison with the binding of sIgA. However, when pilus- 1 is expressed, the binding of serum IgA to the bacterial cell surface is similar to that of sIgA. This observation was confirmed using the nonpiliated $23 \mathrm{~F}$ parental strain and its isogenic pilus-1-knockin mutant. When pilus-1 was expressed, Spn bound higher amounts of human $\operatorname{sIgA}$ (Figure 5B). Surprisingly, pilus-1 seems to be the main pneumococcal determinant capable of binding large amounts of human sIgA. Furthermore, due to the recruitment of serum IgA, binding is not specific to secretory component, suggesting that binding is mediated by the Fab portion and is independent of the secretory component or the J chain only present in sIgA. We confirmed this interpretation by performing binding studies with cleaved sIgA using WT Spn and the $p s p C$-deficient mutant as control. Binding of the sIgA Fab portion to Spn was maintained after cleavage with recombinant IgA1 protease (Figure 5, C and D). In contrast, acquisition of the heavy chain comprising the Fc portion, the secretory component, and the J chain was significantly reduced after protease treatment (Figure 5, C and E). The residual binding of the heavy chain was mediated entirely by PspC. These findings reveal an immunodominant antigen-antibody interaction between pneumococcal pilus- 1 and naturally acquired human sIgA.

In addition, we analyzed the contribution of pilus-1 in Spn binding of purified human lactoferrin and pooled serum IgG, 2 other components of nasal secretions. As shown in Figure 2D, pilus-1 is not involved in recruitment of lactoferrin or IgG to the Spn surface (Figure 5, F and G). Our findings confirm PspA as the main surface protein responsible for binding of lactoferrin.

sIgA is not the only factor required for pneumococcal adherence to $h N F$. As well as assessing the binding of Spn to pooled hNF, we tested pilus-1-mediated Spn adherence to hNF from 6 individual healthy adult donors. Spn adhered to all tested hNF samples 

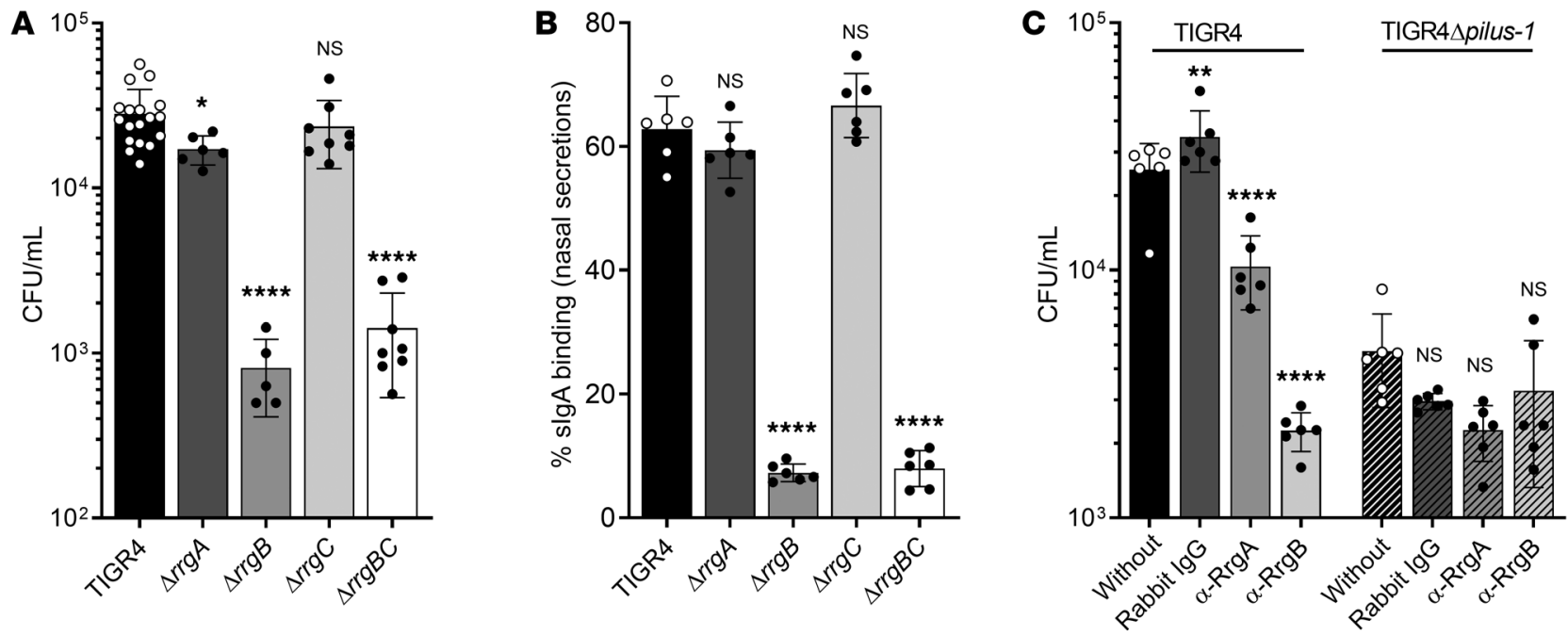

Figure 4. Pilus-1 component RrgB mediates pneumococcal adherence and binding to slgA. (A) Adherence of WT Spn and isogenic mutants deficient for 1 or 2 pilus- 1 components to hNF was assessed in a solid-phase assay. Each bacteria $\left(2 \times 10^{4}\right.$ per $100 \mu \mathrm{L}$ DMEM) were incubated with $10 \mu \mathrm{g}$ hNF in the presence of $0.1 \%$ BSA for 2 hours at $30^{\circ} \mathrm{C}$. Bound bacteria were determined by resuspension with $0.001 \%$ Triton X-100 following plating on TS agar plates supplemented with $200 \mu \mathrm{g} / \mathrm{mL}$ streptomycin. $n=5-18$. (B) Flow cytometric analysis of slgA binding by WT Spn and isogenic mutants in soluble hNF. Bacteria $\left(5 \times 10^{6} \mathrm{CFU}\right.$ per $\left.50 \mu \mathrm{L}\right)$ were incubated with $50 \mu \mathrm{g} / \mathrm{mL}$ of hNF. Binding of surface-associated slgA was analyzed using a FITC-labeled goat anti-human IgA1 antibody and is shown as the percentage binding. $n=6$. (A and B) Results of at least 3 independent experiments are illustrated as mean values \pm SD. ${ }^{*} P<0.05$, ${ }^{* * *} P<0.0001$ vs. TIGR4 by 1-way ANOVA followed by Dunnett's multiple comparison. (C) Inhibition adherence assay using pilus-1-specific antisera. Bacteria were each pretreated with $5 \mu \mathrm{g} / \mathrm{mL}$ of rabbit control serum or anti-RrgA or anti-RrgB antiserum before incubation with immobilized hNF for 2 hours at $30^{\circ} \mathrm{C}$. Detection of bound bacteria was analyzed as described in $\mathbf{A} . n=6$. Results of 3 independent experiments are illustrated as mean values \pm SD. ${ }^{* *} P<0.01,{ }^{* * *} P<0.0001$ vs. without by 1-way ANOVA followed by Šidák's multiple comparison.

in a pilus-1-dependent manner (Figure 6A). We analyzed further whether all hNF samples contain anti-pilus sIgA and verified the presence of anti-RrgB sIgA within the hNF from individual adults (Figure 6B). Using purified sIgA as competitor for Spn binding to hNF showed that adherence of WT Spn, but not the pilus-1-deficient mutant, was inhibited (Figure 6C). This effect was greater for sIgA than an equivalent titer of serum IgA. Due to the inhibitory effect of $\operatorname{sIgA}$, these findings suggest that the sIgA-pilus-1 interaction plays the main role in Spn binding to hNF. Furthermore, we immobilized purified sIgA and BSA (same protein concentration as hNF) and performed the solid-phase assay. WT Spn did not adhere to pilus-1-specific sIgA, suggesting that sIgA alone is not sufficient to mediate adherence (Figure 6D).

Human nasal fluid agglutinates pilus-expressing Spn. Next, we examined whether hNF is able to agglutinate pilus-expressing bacteria. Indeed, we could visualize the formation of immune complexes of piliated Spn but not the pilus-deficient mutant (Figure 7A). The pilus-deficient strain appeared mostly as diplococci and was distributed uniformly in the hNF. In contrast, the pilus-expressing bacteria were more often found associated with mucus particles, in which they form longer chains (threading, an early stage of agglutination) and aggregates (41). Likewise, purified human sIgA (same anti-RrgB titer as hNF) agglutinated Spn in a pilus-dependent manner (Figure 7B).

Nasal mucus forms a highly heterogeneous network in which the mucins generate heterotypic complexes with other mucosal proteins such as SIgA (42). We therefore wanted to check whether the composition and integrity of mucus are necessary for Spn binding, or whether human sIgA alone mediates Spn binding. We centrifuged hNF to sediment large mucus particles and performed the solid-phase assay with the resulting supernatant. We observed that WT Spn adherence was reduced to a level comparable to that of the pilus-1-deficient mutant (Figure 7C). The supernatant of centrifuged hNF still contained sIgA able to agglutinate piliated Spn. However, as shown in Figure 6D, sIgA alone did not mediate Spn adherence. Our results suggest that the interaction between mucus particles and Spn that has been agglutinated by sIgA is necessary for Spn binding to hNF.

Secretory IgA inhibits acquisition of colonization by pilus-expressing pneumococci. A variety of functions of mucosal sIgA have been proposed, including neutralizing released bacterial factors, agglutinating bacteria, and inhibiting bacterial attachment to the epithelium $(22,23,43-47)$. Given the importance of SIgA in protection of the respiratory epithelium, we tested whether human sIgA targeting the type 1 pilus could prevent the establishment of Spn colonization in vivo. Using a mouse model of competitive infection, adult mice were intranasally challenged with equal numbers of the piliated TIGR4 and its isogenic nonpiliated mutant. Before administration, bacteria were preincubated with either a physiologically relevant concentration of human sIgA or PBS as control. Because of the potential of sIgA to disrupt early events in colonization, we determined the competitive index (CI) as early as 4 and 22 hours after infection. In the absence of human sIgA, WT and the nonpiliated mutant established equal levels of colonization (CI approximately equal to 1) (Figure 8, A and C). This indicated a minimal effect of pilus in early colonization in the murine model. When preincubated with human sIgA, which contains naturally acquired antibody against pilus-1, pilus-expressing Spn were 

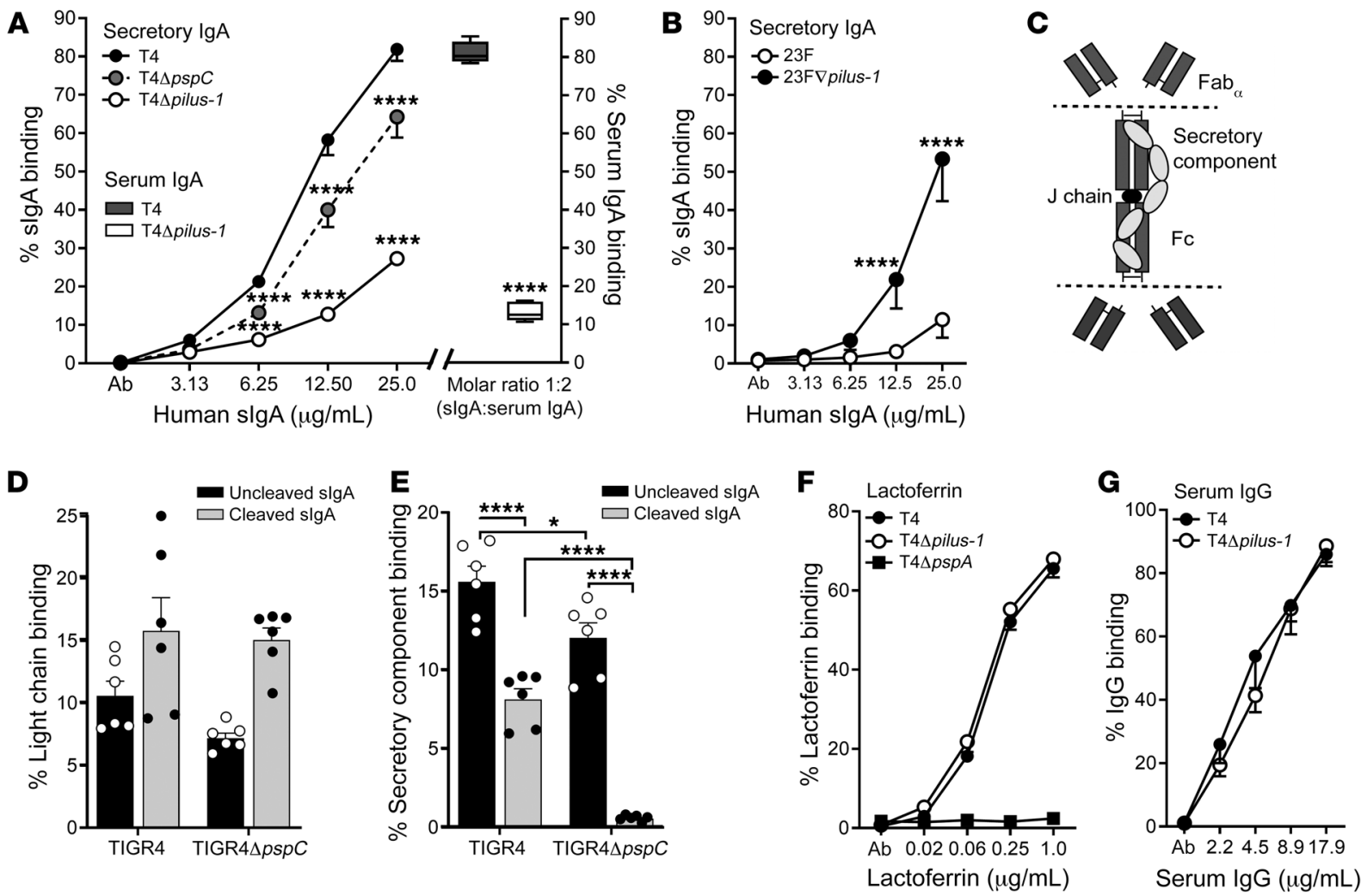

Figure 5. Fab-mediated binding of slgA to pneumococcal type 1 pilus. (A and B) Concentration-dependent binding of soluble purified slgA to Spn. Acquisition of slgA and serum IgA (2-fold molar ratio compared with $25 \mu \mathrm{g} / \mathrm{mL}$ slgA) to type $4 \mathrm{Spn}$ and isogenic mutants (A) or type $23 \mathrm{~F} \mathrm{Spn} \mathrm{and} \mathrm{isogenic}$ pilus-1-knockin mutant (B) was measured by flow cytometry using a specific FITC-labeled goat anti-human IgA1 antibody. The percentage binding of at least 3 independent experiments is shown as mean values with error bars \pm SD. ${ }^{* * *} P<0.0001$ vs. WT by 2-way ANOVA followed by Tukey's multiple comparison, $n=6-8$. (C) Schematic model of cleaved slgA treated with recombinant IgA1 protease (dashed line), generating Fab fragments (binding analyzed in $\mathbf{D}$ ) and Fc fragments with bound secretory component (binding analyzed in $\mathbf{E}$ ). ( $\mathbf{D}$ and $\mathbf{E}$ ) Flow cytometric analysis of slgA binding following cleavage with recombinant IgA1 protease. Binding of slgA light chain (D) or secretory component (E) was analyzed using an anti-human $\kappa$-light chain antibody (D) or a monoclonal anti-secretory component antibody (E). Results of 3 independent experiments are illustrated as mean values with error bars \pm SD. ${ }^{*} P<0.05$, ${ }^{* * * *} P<0.001$ by 1 -way ANOVA followed by Tukey's multiple comparison, $n=6$. ( $\mathbf{F}$ and $\mathbf{G}$ ) Concentration-dependent binding of soluble human lactoferrin and serum IgC to type 4 Spn and isogenic mutants. Results of 3 independent experiments are shown as mean values with error bars \pm SD; $n=6$.

impaired in establishing colonization relative to the nonpiliated mutant at 4 and 22 hours after challenge. The experiment was then repeated using human serum IgA (at a titer to pilus-1 equivalent to sIgA). Serum IgA did not select against the piliated strain, indicating that structural differences in the characteristics of secretory immunoglobulin are needed for this effect (Figure 8A). Additionally, sIgA was pretreated with recombinant IgA1 protease to eliminate its multivalent binding. Protease cleavage, which was confirmed by Western analysis (Figure 8B), was sufficient to eliminate selection against the piliated strain. Since the protease is specific for SIgA1, this result also suggested that any contribution of sIgA2 is secondary. Overall, these findings suggest that naturally acquired sIgA specific to the pneumococcal pilus-1 is able to inhibit colonization acquisition.

Spn expresses a cell surface-anchored protease (Iga, ZmpA) with specificity for human IgA1, the predominant immunoglobulin in nasal secretions $(48,49)$. Cleavage of human IgA1 in the hinge region generates monovalent $\mathrm{Fab}_{a}$ fragments, eliminating its capacity to agglutinate its target $(50,51)$. This would predict that the IgA1 protease would limit agglutination-dependent adherence. When tested in the solid-phase assay with hNF, however, the protease-deficient mutant showed slightly decreased rather than increased adherence (Figure 8D). This observation correlates with a previous report showing that IgA1-mediated adherence of Spn to epithelial cells is enhanced by the enzyme (52). In addition, absence of the IgA1 protease had no effect on immune exclusion by human sIgA in the competition assay in vivo (Figure $8 \mathrm{E}$ ).

Pilus-expressing pneumococci preferentially colonize children. Given our results in vitro and in mice, we hypothesized that human adults, who have had more cumulative exposure to Spn, will have higher levels of mucosal anti-pilus sIgA. As a result, piliated strains may be preferentially excluded during the establishment of adult carriage, and thereby be less commonly found in comparison with carriage in children from the same population.

To test this hypothesis, we analyzed genomic data produced from Spn isolates taken from asymptomatic carriage episodes in unvaccinated mothers and their children (53). We tested for an association between the presence of pilus in the colonizing strain and whether the host was an infant or an adult. We excluded infants under 6 months of age because of maternal antibody. In a naive association, we found adults were less likely to be colonized by pilus-expressing strains, which was significant at the genome- 

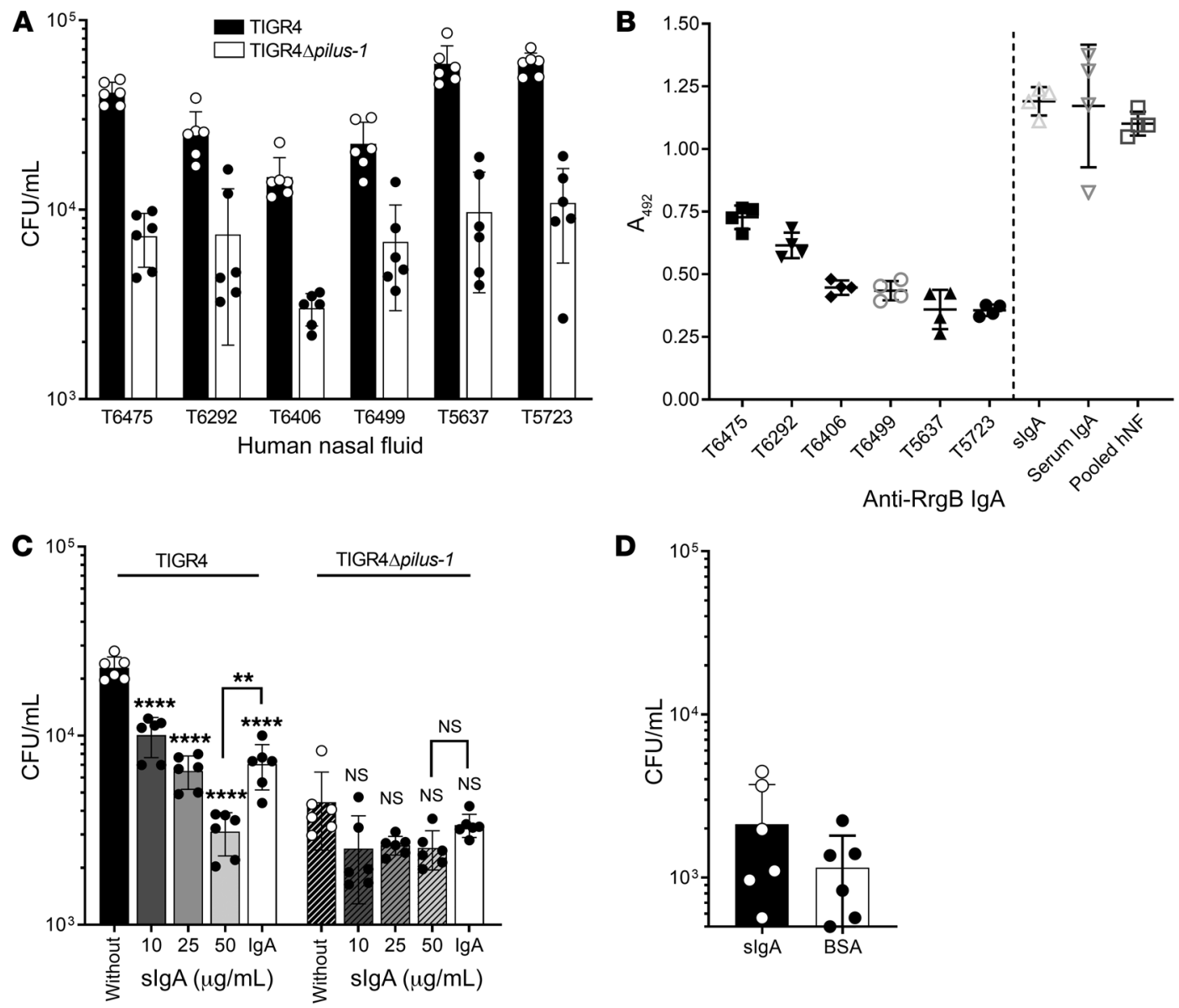

Figure 6. Secretory IgA is necessary but not sufficient for pneumococcal adherence to hNF. (A) Adherence of Spn TIGR4 and isogenic pilus-1-deficient mutant to hNF from 6 individual donors was assessed in a solid-phase assay. Bacteria $\left(2 \times 10^{4}\right.$ per $100 \mu \mathrm{L}$ DMEM) were incubated with $10 \mu \mathrm{g}$ immobilized hNF in the presence of $0.1 \%$ BSA for 2 hours at $30^{\circ} \mathrm{C} . n=6$. (B) Anti-RrgB IgA was determined using an ELISA. Recombinant purified RrgB protein was immobilized in a microtiter plate (Immulon $2 \mathrm{HB}$, Thermo Fisher Scientific) and, after blocking, incubated with $200 \mu \mathrm{g} / \mathrm{mL} \mathrm{hNF}$, or $25 \mu \mathrm{g} / \mathrm{mL} \mathrm{s} / \mathrm{gA}$ and serum IgA, respectively. Binding of RrgB-specific IgA was detected using a biotin-labeled anti-human IgA and peroxidase-coupled streptavidin. The values of control wells without hNF or slgA were subtracted from each measured value. Results are illustrated as mean values \pm SD of 2 independent experiments; $n=4$. (C) Inhibition adherence assay using purified slgA in increasing concentrations or purified serum lgA in a 2-fold molar ratio (compared with $50 \mu g / m L$ slgA). Bacteria were pretreated with either sIgA or serum IgA for 30 minutes at $37^{\circ} \mathrm{C}$ before incubation with immobilized pooled hNF for 2 hours at $30^{\circ} \mathrm{C}$. $n$ = 6. (D) Binding of WT Spn to immobilized slgA or BSA. Secretory IgA and BSA (each $10 \mu$ ) were immobilized overnight followed by blocking with $0.1 \%$ BSA and incubation with $2 \times 10^{4}$ per $100 \mu \mathrm{L}$ bacteria for 2 hours at $30^{\circ} \mathrm{C} . n=6$. (A-C) Experiments were performed in duplicate, and mean values of 3 independent experiments are shown with error bars corresponding to SD. ${ }^{* *} P<0.01,{ }^{* * *} P<0.0001$ by 1-way ANOVA followed by Šidák's multiple comparison (C).

wide level (Table $1 ; \mathrm{OR}=2.23 ; P=2.0 \times 10^{-13}$ ). When controlling for the genotypic background of the colonizing strain, as in a genomewide association study, we found no significant association $(P=$ 0.50). Given the strong association between pilus presence and specific strains, this latter result is unsurprising (54).

\section{Discussion}

We examined the interactions of a mucosal pathogen with mucus-containing human secretions. Our results first confirmed the binding of the Spn surface proteins PspA and PspC to the mucus components lactoferrin and secretory component, respectively $(25,27)$. The dominant interaction of Spn with hNF from adults, however, involved the binding of naturally acquired specific sIgA to a single antigen, the main pilus-1 subunit, RrgB. Binding of sIgA to Spn expressing pilus-1 promoted agglutination and the association of bacterial aggregates (immune complexes) with mucus particles.
In a mouse model of upper respiratory tract colonization, pretreatment with human sIgA enhanced rapid pilus-1-dependent Spn clearance. Thus, our findings provide an antigen-specific demonstration of mucosal defense with natural antibody via immune exclusion; the consecutive events of agglutination, mucus entrapment, and removal of pathogens by mucociliary activities (22).

It was unexpected that a single antigen could account for the majority of Spn adherence to mucus-containing secretions. Pilus-1 is one of many immunogenic surface components but is expressed by only a minority of Spn isolates (less than $20 \%$ in adults from the population we analyzed) $(31,33,35)$. This suggests that the pilus locus is subject to negative frequency-dependent selection, where the population-wide fitness advantage is higher the rarer the locus. It has been shown that negative frequency-dependent selection on genes present in only a minority of the population is a major driving force of pneumococcal population dynamics (55). Our work gives 
A $T 4$

Mucus : Alcian blue

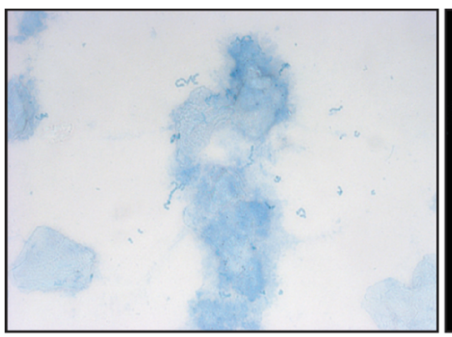

T4 $\Delta$ pilus-1

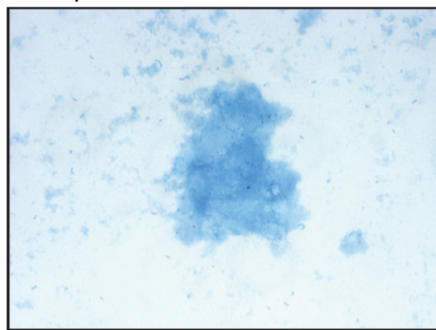

B
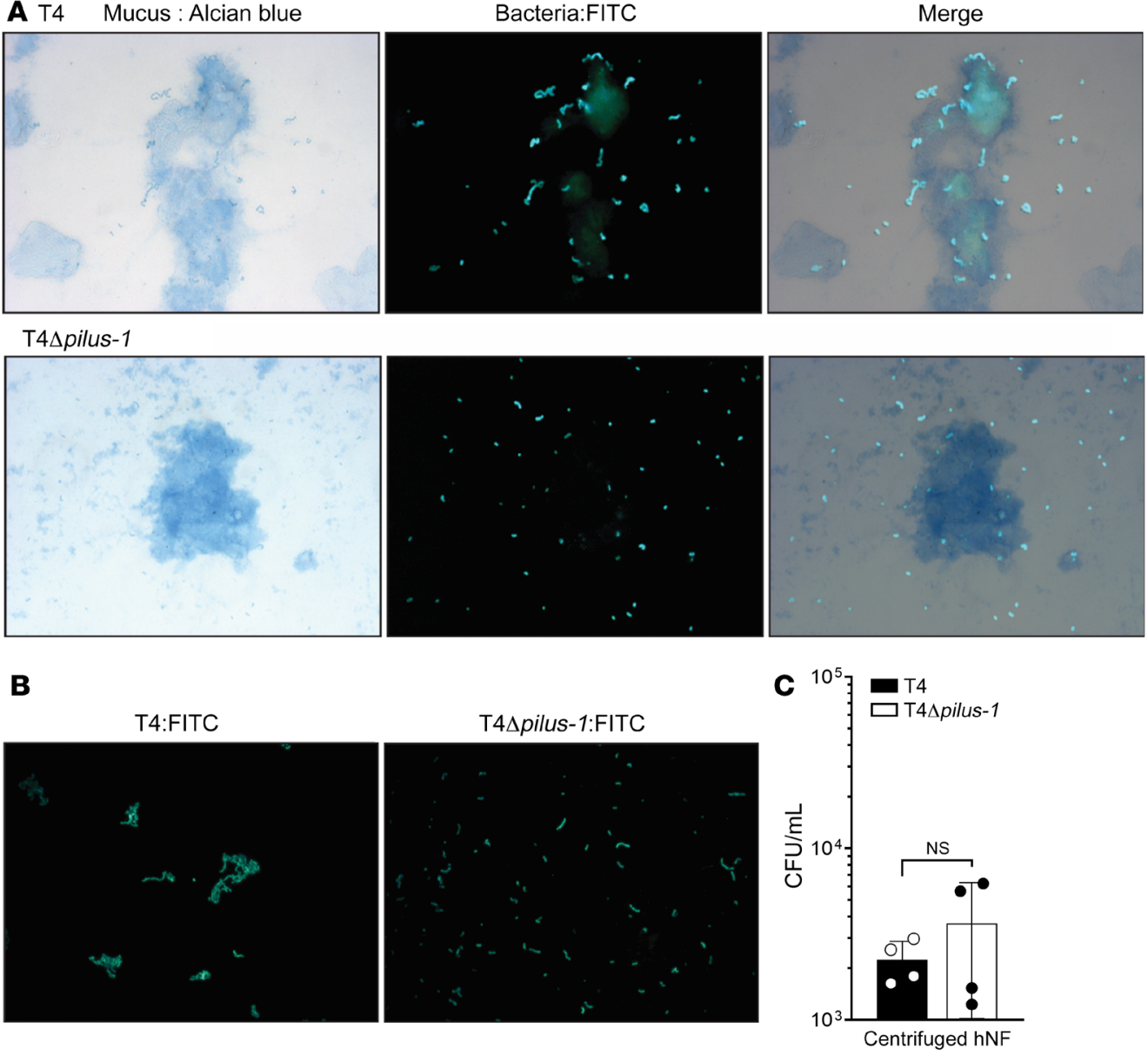

Figure 7. Human nasal fluid agglutinates pneumococci in a pilus-dependent manner. (A and

B) WT Spn and the pilus-1-deficient mutant were incubated with hNF (A) or purified human slgA

(B) for 2 hours at $37^{\circ} \mathrm{C}$ and $5 \%$ $\mathrm{CO}_{2}$. Mucus (blue) was stained with Alcian blue, and bacteria (green) were detected using rabbit anti-capsule antibody and secondary FITC-coupled goat anti-rabbit IgG. Agglutination was visualized by microscopy on an Axiovert 40 CFL microscope equipped with an Axiocam IC digital camera. Original magnification $\times 630$. (C) Adherence of Spn to supernatant of centrifuged hNF. Before immobilization, hNF was centrifuged for 5 minutes at $10,000 \mathrm{~g} ; n=4$ an example of mechanism for this important form of selection, whereby immune exclusion of piliated strains mediated by $\operatorname{sgA}$ would increase as population prevalence of pilus-1 increases.

Naturally acquired immunoglobulin against other surface structures, including the main surface antigen capsular polysaccharide, is common in adults $(30,34,54)$ but, on the basis of our findings, apparently does not contribute in a significant manner to the association of Spn with human nasal mucus. More than $70 \%$ of sIgA binding to Spn was attributable to pilus-1 (Figure 2A and Figure $5 \mathrm{~A})$. In contrast to other Spn surface components, pilus-1 protrudes up to $1 \mu \mathrm{m}$ from the cell surface, where it is no longer shielded by the thick layer of capsular polysaccharide (26). Thus, the physical properties of pilus-1 may allow for binding of sIgA against $\operatorname{RrgB}>>\operatorname{RrgA}$ that was generated through exposure during prior carriage events.

Up to $90 \%$ of mucosal sIgA in human nasal secretions is of the subclass IgA1. Like many successful respiratory pathogens, Spn expresses a protease with specificity for the hinge region of IgA1 to subvert the protective functions of sIgA1. Previous studies have shown that Spn cleaves anti-capsule IgA1/sIgA1, thereby abrogating the agglutinating and opsonophagocytic properties of $\operatorname{sigA} 1(49,56,57)$. The long protrusion of the pilus could explain why there was no detectable effect of the Spn IgA1 protease in limiting immune exclusion. The protease is associated with the cell surface, where it might not access sIgA1 bound to the extended pilus (48).
Secretory IgA is the most abundant immunoglobulin on mucosal surfaces and plays a critical role in the first line of host defense by protecting the underlying epithelium from invading pathogens. The protective function of SIgA is often attributed to its blocking of adherence to cellular receptors or neutralization of secreted toxins, enzymes, or virulence factors via Fab-mediated binding (22). In our mouse model, however, there was no apparent contribution of pilus-1 to adherence, since early colonization of pilus-expressing and that of non-pilus-expressing strains were equivalent, suggesting a human-specific benefit. Therefore, antipilus-1 sIgA does not act by impeding an adhesive function but rather acts through its agglutinating activity. Secretory IgA, which is polymeric (quadrivalent), is more effective at agglutinating piliated Spn compared with serum IgA, which is primarily monomeric (divalent). Several reports document the protective role of secretory polymeric immunoglobulin against mucosal pathogens (5860). The importance of the agglutination function of anticapsular human immunoglobulin in blocking establishment of colonization has previously been documented in a mouse model of carriage following systemic administration of high levels of purified type-specific antibody, and in experimental human pneumococcal carriage following immunization with pneumococcal conjugate vaccine $(56,61)$. In the current study involving naturally acquired mucosal antibody, we demonstrate that sIgA-mucus interaction is necessary for efficient binding to hNF and subsequent removal of agglutinated bacteria. There is evidence that sIgA specifically interacts 
A

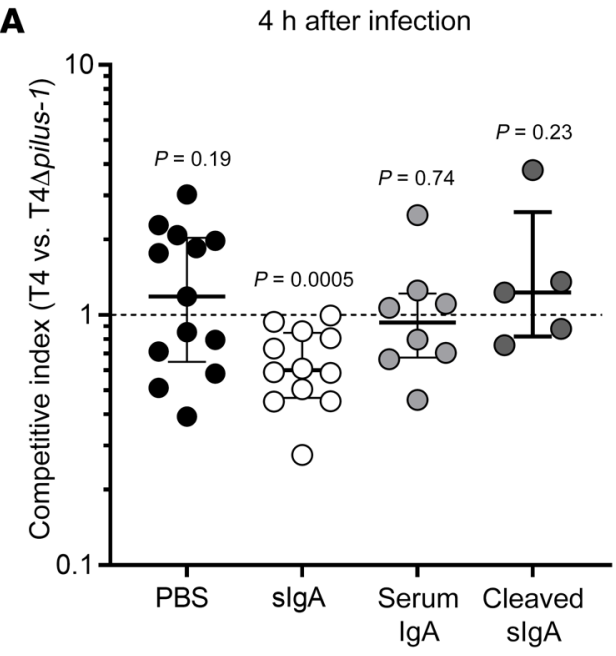

B

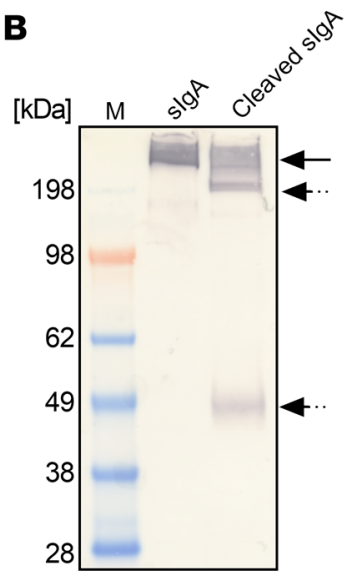

C

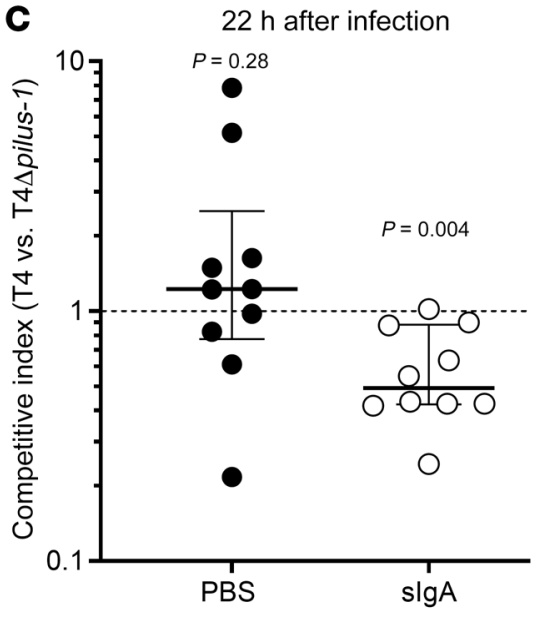

D

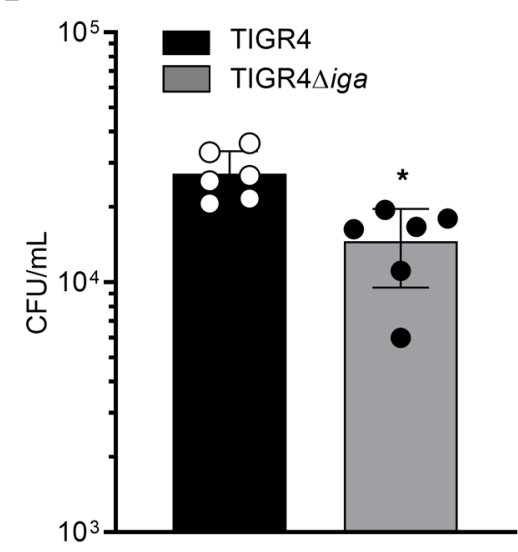

E

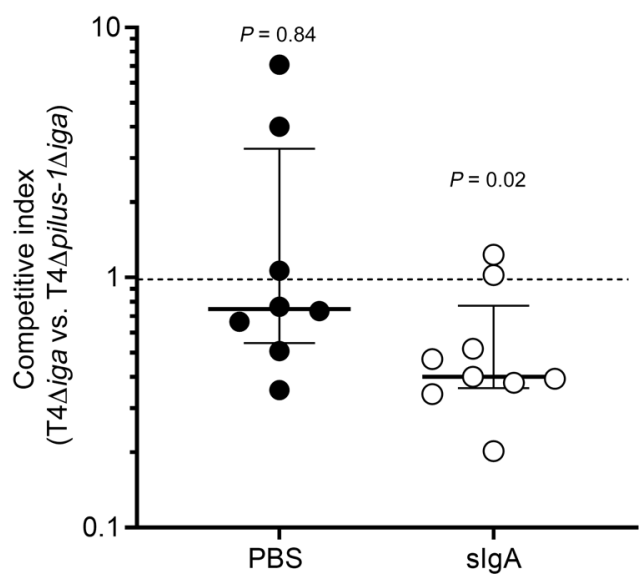

Figure 8. Immune exclusion by pilus-1-specific sIgA. (A-C) Adult mice were intranasally infected with a suspension containing equal amounts of TIGR4 and isogenic pilus-1-deficient mutant. Before administration, bacteria were preincubated with either slgA, serum IgA (equal anti-RrgB titer compared with slgA), cleaved slgA, or PBS. Colonization density and competitive index (CI) were assessed 4 hours (A) and 22 hours (C) after infection by culture of upper respiratory tract lavages followed by colony immunoblot using an anti-RrgB antibody. $n=5-13$. (B) Before administration, slgA was cleaved with recombinant IgA1 protease for 20 hours at $37^{\circ} \mathrm{C}$. Cleavage was visualized on a denaturing and nonreducing SDS-PAGE and Western blot. Solid arrow indicates uncleaved slgA (400 kDa), and dotted arrows indicate cleaved slgA1 (200 kDa and $50 \mathrm{kDa}$ ). (D) Adherence of Spn TIGR4 and isogenic iga-deficient mutant to hNF was analyzed in a solid-phase assay as described before. Results are shown as mean values of 3 independent experiments with error bars corresponding to SD. ${ }^{*} P<0.05$ by 2 -tailed unpaired $t$ test, $n=6$. (E) Adult mice were challenged intranasally with mutants lacking the IgA1 protease. CI was determined as described in C. $n=8-9$. (A, C, and E) Experiments were repeated twice, and groups represent $n=5-13$ animals with median $\pm I Q R$. Dashed lines represent $\mathrm{Cl}=1$. Group medians were compared with a $\mathrm{Cl}=1$ by Wilcoxon signed rank test, and resulting $P$ values are indicated.

with mucins via the mucin-like hinge region of IgA1 and/or though the hydrophilic secretory component (62). In this regard, secretory component ensures the appropriate localization of sIgA within the mucus and thereby contributes to sIgA-mediated immune exclusion. This has been shown in the case of Shigella flexneri infection of the murine gut mucosa using a monoclonal antibody modified to resemble sIgA (58). Alternatively, larger particles generated by agglutination together with their physical association with viscous mucus could be more efficiently swept away and cleared from the nasal surfaces by the mechanics of normal mucociliary flow.

Our study demonstrates protection against Spn by natural secretory immunoglobulin. Our observations also raise the question of whether PspC-secretory component binding is beneficial for the bacterium or aids in mucus-mediated host defense. Secre- tory component is present in the mucus in free form or bound to sIgA/sIgM. Spn binding of secretory component attached to sIgA via PspC might limit Fab-dependent recognition that leads to agglutination and clearance.

Spn colonization is observed predominantly in children younger than 5 years of age, in contrast to much lower carriage rates in healthy adults $(9,10)$. It is apparent that the upper respiratory tract of adults is a less advantageous niche for Spn, perhaps because of the presence of mucosal host defense molecules such as sIgA. In contrast to other immunoglobulins, sIgA production starts late during childhood and reaches adult levels in saliva around 7 years of age (63). The natural development of adaptive immunity against immunogenic pneumococcal proteins reaches its maximum at 3-5 years of age, although these observations are 


\section{Table 1. Proportion of piliated strains in human infants and mothers, based on asymptomatic carriage episodes in an unvaccinated host}

\begin{tabular}{lccc} 
& Nonpiliated & Piliated & \% Piliated \\
Infants (6-24 months) & 1058 & 598 & 56.5 \\
Mothers & 438 & 79 & 18.0 \\
\hline
\end{tabular}

based on serum IgG levels (64). Likewise, anti-pilus antibodies are commonly found in humans, and reach maximum levels around 10-15 years of age (31). Previous studies have tested for an association between pilus-expressing isolates and age of the carriers in vaccinated populations, using either pilus-specific PCR or wholegenome sequencing to perform genotyping, but have found conflicting results $(33,35,65)$. Our results from a large unvaccinated mother-infant cohort showed that pilus-expressing pneumococci were more commonly found in colonized children (after maternally derived immunoglobulin wanes), when antigen-specific mucosal sIgA levels would be low. Indeed, the higher prevalence of piliated strains in children suggests a colonization benefit conferred by pilus- 1 in naive hosts that is diminished later in life, presumable as a result of higher levels of specific mucosal sIgA. This effect itself could contribute to higher carriage rates in young children. However, the lack of independent association when controlling for genetic background means we cannot determine whether this effect is causally driven by the pilus itself or other genetic features on the background of piliated strains. Our experimental studies, however, were carried out with isogenic strains that control for the effect of strain background.

In summary, our study suggests that augmenting mucosal immunity, particularly against the pilus- 1 shaft component $\operatorname{RrgB}$, might accelerate the protection of children, who have the highest carriage rates and burden of disease due to Spn. Additionally, we demonstrate how naturally acquired antibody enhances bacteria-mucus interactions that promote mucosal defense. Finally, we show how these effects of mucosal antibody may drive pathogen adaptation in the natural host among different populations.

\section{Methods}

Ethics statement. All animal experiments in this study followed the guidelines outlined by the National Science Foundation Animal Welfare Act and the Public Health Service Policy on the Humane Care and Use of Laboratory Animals. The Institutional Animal Care and Use Committee (IACUC) at the New York University School of Medicine oversees the welfare, well-being, and proper care and use of all animals. It approved the protocols used in this study.

Chemicals, reagents, and antibodies. Pooled or individual human nasal secretion samples from adult volunteers were purchased from LeeBio. Secretory IgA from human colostrum (I1010), human serum IgA (I4036), human serum IgG (I2511), human lactoferrin, bovine serum albumin (BSA), alkaline phosphatase-coupled goat anti-rabbit IgG (A3687), rabbit anti-human lactoferrin antibody (L-3262), alkaline phosphatase goat anti-human IgA antibody (A-3063), FITC-conjugated goat anti-human IgG (F3512), goat anti-human $\kappa$-light chain antibody (K-0628), FITC-conjugated goat anti-rabbit IgG (F1262), goat anti-human IgA coupled with biotin (B-1015), and Alcian blue solution were obtained from Sigma-Aldrich. Rabbit anti-pneumococcus type 4 serum (16747) was obtained from Statens Serum Institut. Tween-20 and Triton X-100 were obtained from Amresco. Polyclonal rabbit anti-human lysozyme antibody (A0099) was purchased from Dako. FITC-labeled goat anti-human IgA1 (A18782) was obtained from Invitrogen. Allophycocyanin-conjugated (APC-conjugated) goat anti-rabbit IgG (A10931), APC-conjugated anti-mouse IgG (A865), HRP-coupled streptavidin (21130), 5-bromo-4-chloro-3-indolylphosphate/nitro blue tetrazolium (BCIP/NBT) solution, $4 \%$ paraformaldehyde solution (in PBS), and DMEM were obtained from Thermo Fisher Scientific. Mouse monoclonal anti-secretory component antibody (LS-C45754) was purchased from LSBio. FITC-conjugated mouse anti-goat antibody (sc-2356) was purchased from Santa Cruz Biotechnology. Recombinant IgA protease from Neisseria gonorrhoeae was obtained from MoBiTec. Bradford reagent was obtained from BioRad. Protease inhibitor cocktail tablets were purchased from Roche.

Bacterial cultivation. Spn strains used in this study are listed in Table 2. Pneumococci were grown statically in tryptic soy (TS) broth (BD) or in semisynthetic medium $(\mathrm{C}+\mathrm{Y}, \mathrm{pH} 6.8)$ at $37^{\circ} \mathrm{C}$ to an optical density of 0.6 at $620 \mathrm{~nm}\left(\mathrm{OD}_{620}\right)$ for all in vitro experiments, or until an optical density of 1.0 for in vivo colonization of mice. Alternatively, pneumococcal strains were cultivated on TS agar plates supplemented with $100 \mu \mathrm{L}$ of catalase $(30,000 \mathrm{U} / \mathrm{mL}$; Worthington Biomedical) and appropriate antibiotics $(200 \mu \mathrm{g} / \mathrm{mL}$ streptomycin, $250 \mu \mathrm{g} / \mathrm{mL}$ kanamycin, or $2 \mu \mathrm{g} / \mathrm{mL}$ chloramphenicol) overnight at $37^{\circ} \mathrm{C}$ and $5 \% \mathrm{CO}_{2}$.

Recombinant E. coli M15 strains were grown on Luria-Bertani (LB) agar plates supplemented with $100 \mu \mathrm{g} / \mathrm{mL}$ ampicillin at $30^{\circ} \mathrm{C}$ or in liquid LB medium to mid-log phase $\left(\mathrm{OD}_{600}=0.8\right)$ at $200 \mathrm{rpm}$ on an environmental shaker.

Bacterial strains and mutant construction. The primers used for the construction of Spn mutants are summarized in Table 3. In-frame and unmarked deletion mutants of the pneumococcal serotype 4 strain, TIGR4, deficient for the genes $\operatorname{rrg} A, \operatorname{rrgB}, \operatorname{rrg} C, \operatorname{rrgBC}$, or $\operatorname{cbp} A$ were obtained by generation of a PCR product containing a previously described insertion of the "sweet Janus" cassette (66). Deletion of $\operatorname{rrgA}$ and $\operatorname{rrgB}$ was confirmed by dot blots with immunological detection using anti-RrgA and anti-RrgB sera. Serotype 9 and 19F pneumococci lacking the rlrA pathogenicity island (pilus-1) were constructed by transformation with genomic DNA isolated from strain P2454 (TIGR4 $\Delta$ pilus-1) followed by selection on TS agar plates supplemented with $2 \mu \mathrm{g} / \mathrm{mL}$ chloramphenicol and 2 rounds of back-transformation. A pilus-insertion mutant of serotype $23 \mathrm{~F}$ was obtained by transformation of genomic DNA of strain P2535 (TIGR4 $\operatorname{rrgA::Janus)~followed~}$ by selection on TS agar plates containing $250 \mu \mathrm{g} / \mathrm{mL}$ kanamycin followed by 2 rounds of back-transformation. The Janus insertion within the $\operatorname{rrg} A$ gene was replaced with a PCR product of the pilus- 1 islet with selection for streptomycin resistance and screening for acquisition of kanamycin-sensitivity. The expression of pilus- 1 of the serotype $23 \mathrm{~F}$ pneumococci was confirmed via dot blot as described above. The $p s p A$-deficient mutants were obtained by transformation of chromosomal DNA from TIGR4 $\triangle p s p A$ (collection from Marc Lipsitch, Harvard School of Public Health, Boston, MA, USA) or 23F $\Delta p s p A$ (67) into P2406 and P2499, respectively, followed by selection on $200 \mu \mathrm{g} /$ $\mathrm{mL}$ spectinomycin and streptomycin. The iga-deletion mutant was generated by transformation of chromosomal DNA from a previously described construct into P2499 (68). 


\section{Table 2. Bacterial strains and plasmids used in the study}

\begin{tabular}{|c|c|c|c|}
\hline Strain or plasmid & Sero- and genotype & Antibiotic resistance & Source \\
\hline \multicolumn{4}{|l|}{ S. pneumoniae } \\
\hline P2406 (TIGR4) & 4 , clinical isolate & Strep ${ }^{r}$ & Ref. 80 \\
\hline P2422 & TIGR4 $\Delta$ cps & $\operatorname{Kan}^{r}$ & Ref. 80 \\
\hline P2454 & TIGR4 $\Delta$ pilus-1 ( $\Delta$ pilus-1::[m) & Strep', $\mathrm{Cm}^{r}$ & Ref. 80 \\
\hline P2542 & TIGR4 $\triangle r r g A$ & Strep ${ }^{r}$ & This study \\
\hline P2592 & TIGR4 $\Delta r r g B$ & Strep ${ }^{r}$ & This study \\
\hline P2593 & TIGR4 $\Delta r r g[$ & Strepr & This study \\
\hline P2594 & TICR4 $\triangle r r g B C$ & Strep ${ }^{r}$ & This study \\
\hline P2502 & TIGR4 $\Delta p s p A$ & $\operatorname{Kan}^{r}$ & This study \\
\hline P2583 & TIGR4 $\Delta p s p C$ & Strep ${ }^{r}$ & This study \\
\hline P2615 & TIGR4 $\Delta i g a$ & Strep ${ }^{r}$ Spec ${ }^{r}$ & This study \\
\hline P21/P2568 & 9V, clinical blood isolate & & This study \\
\hline P2569 & 9V $\Delta$ pilus-1 ( $\Delta$ pilus-1:: $: \mathrm{m})$ & $\mathrm{Cm}^{r}$ & This study \\
\hline P1860 & 19F, clinical isolate & & This study \\
\hline P2572 & 19F $\Delta$ pilus-1 ( $\Delta$ pilus-1::[m) & $C \mathrm{~m}^{r}$ & This study \\
\hline P2499 & 23F, clinical isolate & Strep ${ }^{r}$ & Ref. 81 \\
\hline P2588 & 23FVpilus-1 (pilus-1 insertion) & Strep' & This study \\
\hline P2617 & 23FVpilus-1 1 iga & Strep', Kanr & This study \\
\hline P2618 & 23F $\Delta p s p A$ (pspA::Spec) & Strep ${ }^{r}$, Spec $^{r}$ & This study \\
\hline P2625 & $23 F \Delta p s p[$ & $K^{\prime}{ }^{r}$ & This study \\
\hline P1547 & $6 \mathrm{~A}$, clinical isolate & & Ref. 68 \\
\hline \multicolumn{4}{|l|}{ E. coli } \\
\hline M15 (pREP4) & 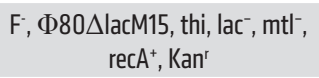 & Kan' & Qiagen \\
\hline
\end{tabular}

\section{Plasmids}

pQE30-rrgB

$A m p r$

Ref. 73

ci were quantified by plating in triplicate on TS agar plates supplemented with appropriate antibiotics and incubated overnight at $37^{\circ} \mathrm{C}$ and $5 \% \mathrm{CO}_{2}$.

For protein cleavage and carbohydrate oxidation, immobilized human mucus was treated for 30 minutes with $50 \mu \mathrm{g} / \mathrm{mL}$ and $100 \mu \mathrm{g} / \mathrm{mL}$ of trypsin (with and without protease inhibitor), or $100 \mathrm{mM}$ sodium periodate $\left(\mathrm{NaIO}_{4}\right)$ in $50 \mathrm{mM}$ sodium acetate buffer (pH 4.5), followed by blocking with $0.1 \%$ BSA/ DMEM as described above. Mild periodate concentration $\left(1 \mathrm{mM} \mathrm{NaIO}_{4}\right)$ leads to selective oxidation of terminal sialic acid, whereas higher concentration of $\mathrm{NaIO}_{4}$ (up to $100 \mathrm{mM}$ ) oxidizes vicinal hydroxyl groups of internal monosaccharides (70-72).

In inhibition assays, pneumococci were preincubated with $5 \mu \mathrm{g} / \mathrm{mL}$ of anti-RrgA, anti-RrgB, and control serum, or increasing concentrations of sIgA $(10-50 \mu \mathrm{g} / \mathrm{mL})$, or serum IgA in a 2-fold molar ratio (in relation to $50 \mu \mathrm{g} / \mathrm{mL}$ sIgA), for 30 minutes at $37^{\circ} \mathrm{C}$ and $5 \% \mathrm{CO}_{2}$.

Flow cytometric analysis. The binding of soluble hNF as well as purified sIgA, human serum IgA, lactoferrin, and human serum IgG to viable pneumococci was analyzed by using flow cytometry. In brief, $5 \times 10^{6}$ bacteria were incubated with either 50 $\mu \mathrm{g} / \mathrm{mL}$ human nasal secretions, increasing concentrations of human sIgA $(0-25 \mu \mathrm{g} / \mathrm{mL})$, serum IgA in a molar ratio of $1: 2$ compared with $25 \mu \mathrm{g} / \mathrm{mL} \operatorname{sigA}$, or increasing concentrations of lactoferrin (0-1.0 $\mu \mathrm{g} / \mathrm{mL})$ and serum $\operatorname{IgG}(0-17.9 \mu \mathrm{g} / \mathrm{mL})$ for $30 \mathrm{~min}$ utes at $37^{\circ} \mathrm{C}$ and $5 \% \mathrm{CO}_{2}$ in 96 -well plates (U-bot-

In vitro proteolytic cleavage of human IgA1. Human sIgA and human nasal secretions were proteolytically cleaved with recombinant IgA protease from $N$. gonorrhoeae. The digestion occurred in reaction buffer (50 mM Tris, $100 \mathrm{mM} \mathrm{NaCl}, 1 \mathrm{mM}$ EDTA, pH 7.5) for 20 hours at $37^{\circ} \mathrm{C}$ with an enzyme/protein ratio of $1: 50$ (wt/wt). Cleavage of IgA was confirmed by Western blot and anti-human IgA conjugated to alkaline phosphatase.

Human nasal secretion-binding assay. Adherence of different pneumococcal strains to human nasal fluid (hNF) was assessed in a solidphase binding assay as previously described $(13,69)$. To generate homogeneous samples, hNF samples were sonicated for 1 second with an amplitude of 10. In brief, nasal mucus or protease-treated mucus $(10 \mu \mathrm{g} /$ well $)$ was immobilized in PBS in a 96-well flat-bottom plate (Sarstedt) followed by centrifugation at $250 \mathrm{~g}$ for 3 minutes and incubation overnight at $37^{\circ} \mathrm{C}$. The plates were gently washed 3 times with DMEM medium, and the wells were blocked with 0.1\% BSA/DMEM for 2 hours at room temperature. Spn cultures were grown to mid-log phase $\left(\mathrm{OD}_{620}=0.6\right)$ and diluted in DMEM. After washing of the plate with DMEM, a bacterial suspension of $2 \times 10^{4}$ bacteria in $100 \mu \mathrm{L}$ was applied to the wells by centrifugation at $250 \mathrm{~g}$ for 3 minutes at room temperature. Interaction with hNF was allowed for at least 2 hours and up to 5 hours at $30^{\circ} \mathrm{C}$ and $5 \% \mathrm{CO}_{2}$. Unbound bacteria were recovered by collection of the supernatant and gentle washing of the wells 19 times each with $100 \mu \mathrm{L}$ DMEM. Adherent bacteria were lifted by addition of $100 \mu \mathrm{L}$ of $0.001 \%$ Triton X-100/PBS for 15 minutes at $30^{\circ} \mathrm{C}$ and $5 \%$ $\mathrm{CO}_{2}$ followed by vigorous mixing. Unbound and adherent pneumococ- tom; Greiner Bio-One). Likewise, Spn were also incubated with 12.5 $\mu \mathrm{g} / \mathrm{mL}$ of proteolytically cleaved sIgA. After washing twice with $1 \%$ BSA/PBS, binding of human proteins was detected using either FITClabeled goat anti-human IgA1 (1:250), rabbit anti-human lactoferrin $\operatorname{IgG}$ (1:500), or FITC-conjugated goat anti-human IgG (1:250) followed by secondary APC-labeled anti-rabbit IgG (1:100). Antibody incubations occurred in $0.1 \%$ BSA/PBS for 45 minutes at $4^{\circ} \mathrm{C}$. Finally, bacteria were washed and fixed with $2 \%$ paraformaldehyde in PBS at room temperature for 1 hour, and fluorescence intensity was measured using a FACS LSR II flow cytometer and FACSDiva software 8.0.1 (Becton Dickinson) for data acquisition and analysis with FlowJo software 10.3. A gating region was set to exclude bacterial aggregates and debris. The results of human protein binding to pneumococci are shown as the percentage of labeled bacteria.

Heterologous expression and purification of recombinant RrgB protein. Purification of recombinant $\operatorname{RrgB}$ protein used in this study has

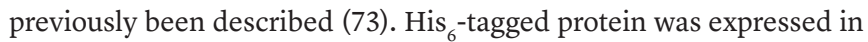
E. coli M15 after induction with $1 \mathrm{mM}$ isopropyl $\beta$-D-thiogalactopyranoside for 4 hours at $30^{\circ} \mathrm{C}$. Overexpressed RrgB protein was purified from $E$. coli lysates under native conditions via immobilized metal affinity chromatography using a HisTrap column and the ÄKTA purifier system according to the manufacturer's instructions (GE Healthcare). Protein was dialyzed (30 kDa molecular weight cutoff) against PBS ( $\mathrm{pH}$ 7.4) at $4^{\circ} \mathrm{C}$ using centrifugal filters (Millipore). Determination of the protein concentration was performed using Bradford reagent, and protein purity was confirmed by Western blot. 


\section{Table 3. Primers used in the study}

Gene target

P2535:

rrgA::Janus-cassette

P254:

P2542:

clean deletion $r r g A$

P2589:

rrgB::Janus-cassette

\section{P2592:}

clean deletion $\mathrm{rrg} B$

\section{P2590:}

rrg[:::Janus-cassette

\section{P2593:}

clean deletion $\mathrm{rrg} C$

P2591:
rrgBC::Janus-cassette
P2594:
clean deletion $r r g B$ and $r r g C$

P2582:

psp[::/anus-cassette

\section{P2583:}

clean deletion $p s p C$

Presence of rlrA pathogenicity island

Absence of rlrA pathogenicity island

\section{Primer name}

$\operatorname{rrg} A$ upstream forward $\operatorname{rrg} A$ upstream reverse rrgA Janus forward

rrgA Janus reverse

rrgA downstream forward

rrgA downstream reverse

$\operatorname{rrgA}$ upstream forward

rrgA upstream reverse

rrgA downstream forward

$\operatorname{rrg} A$ downstream reverse

$\operatorname{rrg} B$ upstream forward

$\operatorname{rrg} B$ upstream reverse

$\operatorname{rrg} B$ Janus forward

$\operatorname{rrg} B$ Janus reverse

$\operatorname{rrg} B$ downstream forward

$\operatorname{rrg} B$ downstream reverse

$\mathrm{rrg} B$ upstream forward

$\operatorname{rrg} B$ upstream reverse

$\operatorname{rrg} B$ downstream forward

$\operatorname{rrg} B$ downstream reverse

rrgC upstream forward

rrgC upstream reverse

$\operatorname{rrg}[$ ]anus forward

$\operatorname{rrg}$ C Janus reverse

$\operatorname{rrg}[$ downstream forward

$\operatorname{rrg}$ C downstream reverse

rrgC upstream forward

rrgC upstream reverse

$\operatorname{rrg}[$ downstream forward

$\operatorname{rrg}[$ downstream reverse

$\operatorname{rrgBC}$ upstream forward

$\operatorname{rrg} B C$ upstream reverse

$\operatorname{rrg} B C$ Janus forward

$\operatorname{rrg} B C$ Janus reverse

$\operatorname{rrg} B C$ downstream forward

$\operatorname{rrg} B C$ downstream reverse

$\operatorname{rrg} B C$ upstream forward

$\operatorname{rrg} B C$ upstream reverse

$\operatorname{rrg} B C$ downstream forward

$\operatorname{rrg} B C$ downstream reverse

$p s p[$ upstream forward

$p s p[$ upstream reverse

$p s p[$ Janus forward

$p s p[$ Janus reverse

$p s p$ C downstream forward

$p s p[$ downstream reverse

$p s p[$ upstream forward

$p s p[$ upstream reverse

$p s p C$ downstream forward

$p s p[$ downstream reverse

RrgB_seq_forw

RrgB_seq_rev1

Pilus1-neg_Forw

Pilus1-neg_Rev
Sequence $\left(5^{\prime} \rightarrow 3^{\prime}\right)$

CGTGTATACAGATTGAAAGTACCTATCAATC

CACATTATCCATTAAAAATCAAACGGAAACCATTATTCATAGAAC

GTTCTATCAATAATGCTTTCCGTTTGATTTTTAATGGATAATGTG

СATTTCTACACTTTACGGTTATGCTTTTGGACGTTTAGTACC

GGTACTAAACGTCCAAAAGCATAACCGTAAAGTGTAGAAATG

GCTACTTCTTAAAAGTATCGTTCAGAAC

CGTGTATACAGATTGAAAGTACCTATGAATC

ATСATTTCTACACTTTACGGATGTTTCCGCTCCCTGTTAAGCATTATTCATAGAACAAG

CTTGTTCTATGAATAATGCTTAACAGGGAGCGGAAACATCCGTAAAGTGTAGAAATGAT

GCTACTTCTTAAAAGTATCGTTCAGAAC

GTGCTCTATGATACGACTGAGAAAAGGATTCG

TCAAACGGATCCСATCCATTTCСTCTTTCATCATTTC

GAAATCATCAAAGAGGAAATGGATCGGATCCGTTTGA

СТСTTACTTAAGCGGGCСССТTTCСTTATGC

GCATAAGGAAAGGGGCCCGCTTAAGTAAGAG

САTACTCTACACCTTCСACTCGGAAACGAC

GTGCTCTATCATACGACTGAGAAAAGCATTCG

СTCTCTTACTTAAGCAAGTTCATCGTTGATTGATTTCATCATTTCTCC

GGAGAAATCATCAAATCAATCAACCATCAACTTGCTTAAGTAAGAGAG

САTACTCTACACCTTCCACTCGGAAACGAC

GCAACGTTCGATTTGGTTAATGCTCAGACTGG

CAAACCGATCCGATCCATTTCCTCAATCATTTTCTCC

GCAGAAAATCATTCAGGAAATGGATCGGATCCGTTTG

CATTGAATATCAGTTGGGCCCCTTTCCTTATGC

GCATAAGGAAAGGGGCCCAACTGATATTCAATG

GGTCACATAATCATGACCTGGTACAATCAATAAATC

GCAACGTTCGATTTGGTTAATGCTCAGACTGG

CATTGAATATCAGTTATTTGGTTTGATACGACTAATCATTTTCTGCAT

ATGCAGAAAATCATTAGTCGTATCAAACCAAATAACTGATATTCAATG

GGTCACATAATCATGACCTGGTACAATCAATAAATC

GTGCTCTATGATACGACTGAGAAAAGGATTCG

TCAAACGGATCСGATCCATTTCСTCTTTCATGATTTC

CAAATCATGAAAGAGGAAATGGATCGGATCCGTTTGA

CATTGAATATCAGTTGGGCCCCTTTCCTTATGC

GCATAAGGAAAGGGGGCCAACTGATATTCAATG

GGTCACATAATCATGACCTGGTACAATCAATAAATC

GTGCTCTATGATACGACTGAGAAAAGCATTCG

CATTGAATATCAGTTATTTGGTTTGTTGATTGATTTCATGATTTCTCC

GGAGAAATCATGAAATCAATCAACAAACCAAATAACTGATATTCAATG

GGTCACATAATCATCACCTGGTACAATCAATAAATC

GGGCATTGGATAAGGTTTTGACAAACCTG

САСАTTATCСATTAAAAATCAAACGGAAAACATGTTTATTTCC

GGAAATAAACATGTTTTCCGTTTGATTTTTAATGGATAATGTG

GTTATATTAGGTTTAGTTCCAGAGACCTGGGCCCCTTTCCTTATGC

GCATAAGGAAAGGGGCCCAGGTCTCTGGAACTAAACCTAATATAAC

CGATATCGTCGATATCAACATGGGCTG

GGGCATTGGATAAGGTTTTGACAAACCTG

TTAGGTTTAGTTTACCCATTCTTTTGATGCAAACATGTTTATTTCC

GGAAATAAACATGTTTGCATCAAAAGAATGGGTAAACTAAACCTAA

CGATATCGTCGATATCAACATGGGCTG

CGAAAACTTGCACAGAAAAAGGATTATTATTGTC

GCTTTGGAGTATTCCCGTCATCTGG

CGCCTTGCATGCATTGAGC

GTATTACAAGATATTATTTCACC 
ELISA. To determine anti-RrgB IgA titers in hNF, colostrum sIgA, and serum IgA, wells of microtiter plates (96-well, Immulon $2 \mathrm{HB}$ plate, Thermo Fisher Scientific) were coated with $0.1 \mu \mathrm{g} /$ well recombinant $\mathrm{RrgB}$ in PBS ( $\mathrm{pH} 7.4$ ) overnight at $4^{\circ} \mathrm{C}$. The plates were washed 3 times with washing buffer (PBS, 0.05\% Tween-20), and blocked with blocking buffer (PBS, 0.1\% Tween-20 supplemented with 1\% BSA) for 1 hour at room temperature. Afterward the washed wells were incubated with $200 \mu \mathrm{g} / \mathrm{mL}$ hNF, $25 \mu \mathrm{g} / \mathrm{mL}$ sIgA, and $25 \mu \mathrm{g} / \mathrm{mL}$ serum IgA in PBS for 1 hour at $37^{\circ} \mathrm{C}$. Bound anti-RrgB IgA was measured using a goat anti-human IgA coupled with biotin and HRP-coupled streptavidin. O-Phenylenediamine dihydrochloride was used as HRP substrate, and color reaction was measured at an absorbance of $492 \mathrm{~nm}$ using a spectraMax M3 reader (Molecular Devices). The values of control wells without IgA were subtracted from each measured value.

Competitive colonization in mice. C57BL/6J mice were purchased from The Jackson Laboratory, and bred and housed in a conventional animal facility as previously described (74). During colonization, all mice appeared healthy and did not experience any weight loss in comparison with uninfected controls. Spn strains were grown to an $\mathrm{OD}_{620}$ of 1.0, washed, and diluted to the desired density in PBS. A mixture containing a 1:1 ratio of the TIGR4 strain and isogenic pilusdeficient mutant was preincubated with either human SIgA (final concentration $0.9 \mathrm{mg} / \mathrm{mL}$ in PBS), human serum IgA, or PBS as control for 30 minutes at room temperature. Five-week-old adult mice were inoculated intranasally without anesthesia with $15 \mu \mathrm{L}$ containing 1.5 $\times 10^{5}$ to $2 \times 10^{5} \mathrm{CFU}$ of the mixed pneumococcal strains. At 4 hours or 22 hours after pneumococcal challenge, mice were euthanized using $\mathrm{CO}_{2}$ followed by cardiac puncture. For quantification of the colonization density, the trachea was cannulated and lavaged with $200 \mu \mathrm{L}$ of sterile PBS, and fluid collected from the nares. The nasal lavage samples were plated in serial dilution on TS agar plates supplemented with $200 \mu \mathrm{g} / \mathrm{mL}$ spectinomycin. To calculate the competitive index (CI), a colony immunoblot detecting the pilus-expressing strain was performed. In brief, cultivated TS agar plates containing the total number of pneumococcal colonies were blotted on circular nitrocellulose membranes. After drying for 15 minutes, the membrane was blocked with 2\% BSA/PBS for at least 3 hours followed by incubation with a primary rabbit anti-RrgB antibody (1:200,000 in $0.1 \%$ BSA/0.01\% Tween-20/PBS) overnight at $4^{\circ} \mathrm{C}$. The membrane was washed 3 times with $0.05 \%$ Tween-20/PBS and incubated with a secondary alkaline phosphatase-coupled anti-rabbit IgG (1:5000 in $0.1 \%$ BSA/0.01\% Tween-20/PBS) for 1 hour at room temperature. The colorimetric visualization of the pilus-expressing pneumococcal colonies occurred by use of BCIP/NBT as substrate. To determine the quantity of the nonpiliated colonies, the number of pilus-expressing colonies was subtracted from the total number of colonies. The CIs were calculated as the ratio of pilus-expressing strain to pilusdeficient mutant output $\mathrm{CFU} / \mathrm{mL}$ divided by the pilus-expressing strain to pilus-deficient mutant input CFU/mL. The colony immunoblots were performed at least in duplicate from at least 2 independent colonization experiments to ensure reproducibility.

Agglutination assay and microscopic visualization. For agglutination, $5 \times 10^{4}$ bacteria were incubated with $10 \mu \mathrm{L}$ of undiluted hNF or purified $\operatorname{sIgA}$ (which contained the same titer of anti-RrgB $\operatorname{IgA}$ ) for 2 hours at $37^{\circ} \mathrm{C}$ and $5 \% \mathrm{CO}_{2}$. Samples were placed onto glass slides and immobilized via heat fixation. To visualize mucus, samples were incubated with $3 \%$ acetic acid followed by incubation with
Alcian blue (in 3\% acetic acid, $\mathrm{pH} 2.5$ ) for 30 minutes. After washing in water for 10 minutes, slides were blocked in $10 \% \mathrm{FBS}$ at $4^{\circ} \mathrm{C}$ overnight. Bacteria were stained with primary rabbit anti-capsule antibody (1:200 in 0.5\% FBS/PBS) and secondary FITC-coupled goat anti-rabbit IgG (1:100 in 0.5\% FBS/PBS). Agglutination was visualized on an Axiovert 40 CFL microscope equipped with an Axiocam IC digital camera (Zeiss). All image analysis was performed with ZEN 2012 software, and images were processed with ImageJ 1.52a (NIH) for brightness and contrast.

SDS-PAGE and Western blot analysis. Western blots were performed to detect proteinaceous components in hNF. One microgram of purified lactoferrin, sIgA, or lysozyme, as well as pooled hNF, was separated under denaturing conditions using 4\%-12\% Bolt Bis-Tris Plus gels (Thermo Fisher Scientific). Separated proteins were transferred on nitrocellulose membranes via a dry blotting system (iBlot 2, Thermo Fisher Scientific). Following blocking of the membrane with 2\% BSA/PBS for at least 3 hours and washing 3 times with PBS/0.05\% Tween-20, proteins were detected with a polyclonal rabbit antihuman lactoferrin antibody (1:10,000), alkaline phosphatase-coupled goat anti-human IgA antibody (1:20,000), or rabbit anti-human lysozyme antibody $(1: 10,000)$ overnight at $4^{\circ} \mathrm{C}$ followed by incubation with an alkaline phosphatase-coupled goat anti-rabbit IgG (1:5000) for 1 hour at room temperature. All antibody incubations occurred in 0.1\% BSA/0.01\% Tween-20/PBS. Protein bands were visualized after washing of the membrane 3 times with $0.05 \%$ Tween-20/PBS using $\mathrm{BCIP} / \mathrm{NBT}$ as substrate.

Association between pilus and age in a human population. We used genomic data from the Mae La refugee camp (75). This consisted of data from around 600 children and their mothers sampled every month from birth to 2 years of age; genomic data associated with 3000 randomly selected positive swabs were available (53). We gave each sample a binary outcome, mothers being positive and infants being negative; infants under 6 months of age were excluded.

To determine pilus presence, we used a definition of the accessory genome in this population and classified any sequence containing at least 1 of the $3 \mathrm{rrgB}$ alleles (cluster of orthologous genes CLSO2709, CLS03842, and CLS01960) as being piliated $(30,55)$. To control for the same isolate being observed multiple times, we assigned a unique identifier to each individual carriage episode; these identifiers have been previously defined using a hidden Markov model (76).

We then used a generalized linear mixed model (LMM) with a Bernoulli error structure and logit link function to test for an association between pilus presence and age. Pilus presence was treated as a fixed effect, and a random intercept for each carriage episode was included. We calculated a $P$ value for the association by using a likelihood-ratio test between this model and a nested model with the pilus term removed.

To calculate this association while also controlling for genetic background, we also used an LMM with the genetic kinship between isolates as random effects, as in genome-wide association studies (77, 78). We used the pyseer package (version 1.2.0) in LMM mode, with the kinship/covariance matrix calculated from a neighbor-joining tree of all genome sequences in the cohort $(53,79)$.

Data availability. Data and code to perform the association between pilus and age can be found on GitHub: https://github.com/johnlees/ pilus-age/commit/d37524f52aa1d4ffadf2bfd0a2bb7cb28f7fd552 (commit ID: d37524f5). 
Statistics. Statistical analysis was performed using GraphPad Prism (version 7.01, GraphPad Software Inc.). Data of in vitro experiments are reported as mean \pm SD. Results from in vivo assays are shown as median with interquartile range. Unless otherwise specified, statistical analyses were performed using $t$ test or 1-way ANOVA with either Šidák's or Dunnett's multiple-comparisons test. A $P$ value less than 0.05 was considered to be statistically significant.

Study approval. The experimental protocols were approved by the IACUC of the New York University School of Medicine.

\section{Author contributions}

UB contributed to project design, performed in vitro and in vivo experiments and data analysis and interpretation, and wrote the manuscript. JAL performed population genomics, interpreted data, and contributed to the writing. AJH constructed $r r g A$ - and $p s p C$-deficient pneumococcal mutants. JNW oversaw the project conception and design, data interpretation, manuscript preparation, and funding acquisition.

\section{Acknowledgments}

We thank Richard Malley and Alan Basset for providing the anti-pilus-1 antibodies and the recombinant E. coli strain. We thank Nicholas Croucher for sharing data defining presence and absence of pilus genes in pneumococcal populations. This project was supported by grants from the US Public Health Service to JNW (AI038446 and AI105168).

Address correspondence to: Jeffrey N. Weiser, Department of Microbiology, New York University School of Medicine, Alexandria Center for Life Sciences - West Tower, 430 East 29th Street, New York, New York 10016, USA. Phone: 212.263.1080; Email: Jeffrey.Weiser@nyulangone.org.
1. Cone RA. Barrier properties of mucus. Adv Drug Deliv Rev. 2009;61(2):75-85.

2. Vareille M, Kieninger E, Edwards MR, Regamey $\mathrm{N}$. The airway epithelium: soldier in the fight against respiratory viruses. Clin Microbiol Rev. 2011;24(1):210-229.

3. Boucher RC. Molecular insights into the physiology of the 'thin film' of airway surface liquid. JPhysiol (Lond). 1999;516(pt 3):631-638.

4. Button B, et al. A periciliary brush promotes the lung health by separating the mucus layer from airway epithelia. Science. 2012;337(6097):937-941.

5. Taherali F, Varum F, Basit AW. A slippery slope: on the origin, role and physiology of mucus. $A d v$ Drug Deliv Rev. 2018;124:16-33.

6. Cole AM, Dewan P, Ganz T. Innate antimicrobial activity of nasal secretions. Infect Immun. 1999;67(7):3267-3275.

7. Travis SM, et al. Activity of abundant antimicrobials of the human airway. Am J Respir Cell Mol Biol. 1999;20(5):872-879.

8. Diamond G, Legarda D, Ryan LK. The innate immune response of the respiratory epithelium. Immunol Rev. 2000;173:27-38.

9. Abdullahi $O$, et al. The prevalence and risk factors for pneumococcal colonization of the nasopharynx among children in Kilifi District, Kenya. PLoS One. 2012;7(2):e30787.

10. Yahiaoui RY, et al. Prevalence and antibiotic resistance of commensal Streptococcus pneumoniae in nine European countries. Future Microbiol. 2016;11:737-744.

11. Weiser JN, Ferreira DM, Paton JC. Streptococcus pneumoniae: transmission, colonization and invasion. Nat Rev Microbiol. 2018;16(6):355-367.

12. Nelson AL, Roche AM, Gould JM, Chim K, Ratner AJ, Weiser JN. Capsule enhances pneumococcal colonization by limiting mucus-mediated clearance. Infect Immun. 2007;75(1):83-90.

13. Zafar MA, Hamaguchi S, Zangari T, Cammer M, Weiser JN. Capsule type and amount affect shedding and transmission of Streptococcus pneumoniae. MBio. 2017;8(4):e00989-17.

14. King SJ, Hippe KR, Weiser JN. Deglycosylation of human glycoconjugates by the sequential activities of exoglycosidases expressed by Streptococcus pneumoniae. Mol Microbiol. 2006;59(3):961-974.
15. Marion C, Limoli DH, Bobulsky GS, Abraham JL, Burnaugh AM, King SJ. Identification of a pneumococcal glycosidase that modifies O-linked glycans. Infect Immun. 2009;77(4):1389-1396.

16. King SJ. Pneumococcal modification of host sugars: a major contributor to colonization of the human airway? Mol Oral Microbiol. 2010;25(1):15-24.

17. King SJ, et al. Phase variable desialylation of host proteins that bind to Streptococcus pneumoniae in vivo and protect the airway. Mol Microbiol. 2004;54(1):159-171.

18. Yesilkaya H, Manco S, Kadioglu A, Terra VS, Andrew PW. The ability to utilize mucin affects the regulation of virulence gene expression in Streptococcus pneumoniae. FEMS Microbiol Lett. 2008;278(2):231-235.

19. Marion C, Burnaugh AM, Woodiga SA, King SJ. Sialic acid transport contributes to pneumococcal colonization. Infect Immun. 2011;79(3):1262-1269.

20. Ha U, et al. A novel role for IkappaB kinase (IKK) $\alpha$ and IKK $\beta$ in ERK-dependent up-regulation of MUC5AC mucin transcription by Streptococcus pneumoniae. JImmunol. 2007;178(3):1736-1747.

21. Zafar MA, Wang Y, Hamaguchi S, Weiser JN. Host-to-host transmission of Streptococcus pneumoniae is driven by its inflammatory toxin, pneumolysin. Cell Host Microbe. 2017;21(1):73-83.

22. Mantis NJ, Rol N, Corthésy B. Secretory IgA's complex roles in immunity and mucosal homeostasis in the gut. Mucosal Immunol. 2011;4(6):603-611.

23. Russell MW, Kilian M, Mantis NJ, Corthésy B. Biological activities of IgA. In: Mestecky J, Strober W, Russell MW, Kelsall BL, Cheroutre H, Lambrecht BN, eds. Mucosal Immunology. 4th ed. Boston, Massachusetts, USA: Academic Press; 2015:429-454.

24. Raphael GD, Baraniuk JN, Kaliner MA. How and why the nose runs. JAllergy Clin Immunol. 1991;87(2):457-467.

25. Hammerschmidt S, Bethe G, Remane PH, Chhatwal GS. Identification of pneumococcal surface protein A as a lactoferrin-binding protein of Streptococcus pneumoniae. Infect Immun. 1999;67(4):1683-1687.

26. Hilleringmann M, et al. Pneumococcal pili are composed of protofilaments exposing adhesive clusters of Rrg A. PLoS Pathog. 2008;4(3):e1000026.

27. Hammerschmidt S, Talay SR, Brandtzaeg P, Chhatwal GS. SpsA, a novel pneumococcal surface protein with specific binding to secretory immunoglobulin A and secretory component. Mol Microbiol. 1997;25(6):1113-1124.

28. Hammerschmidt S, Tillig MP, Wolff S, Vaerman JP, Chhatwal GS. Species-specific binding of human secretory component to SpsA protein of Streptococcus pneumoniae via a hexapeptide motif. Mol Microbiol. 2000;36(3):726-736.

29. Iovino F, et al. pIgR and PECAM-1 bind to pneumococcal adhesins RrgA and PspC mediating bacterial brain invasion. J Exp Med. 2017;214(6):1619-1630.

30. Croucher NJ, et al. Diverse evolutionary patterns of pneumococcal antigens identified by pangenome-wide immunological screening. Proc Natl Acad Sci U S A. 2017;114(3):E357-E366.

31. Ahmed MS, et al. Immune responses to pneumococcal pilus RrgA and RrgB antigens and their relationship with pneumococcal carriage in humans. J Infect. 2014;68(6):562-571.

32. Jiménez-Munguía I, van Wamel WJ, Olaya-Abril A, García-Cabrera E, Rodríguez-Ortega MJ, Obando I. Proteomics-driven design of a multiplex bead-based platform to assess natural IgG antibodies to pneumococcal protein antigens in children. J Proteomics. 2015;126:228-233.

33. Azarian T, et al. Association of pneumococcal protein antigen serology with age and antigenic profile of colonizing isolates. J Infect Dis. 2017;215(5):713-722.

34. Ramos-Sevillano E, Ercoli G, Brown JS. Mechanisms of naturally acquired immunity to Streptococcus pneumoniae. Front Immunol. 2019;10:358.

35. Regev-Yochay G, et al. Re-emergence of the type 1 pilus among Streptococcus pneumoniae isolates in Massachusetts, USA. Vaccine. 2010;28(30):4842-4846.

36. Moschioni M, et al. Streptococcus pneumoniae contains 3 rlrA pilus variants that are clonally related. JInfect Dis. 2008;197(6):888-896.

37. Aguiar SI, Serrano I, Pinto FR, Melo-Cristino J, Ramirez M. The presence of the pilus locus is a 
clonal property among pneumococcal invasive isolates. BMC Microbiol. 2008;8:41.

38. LeMieux J, Hava DL, Basset A, Camilli A. RrgA and $\mathrm{RrgB}$ are components of a multisubunit pilus encoded by the Streptococcus pneumoniae rlrA pathogenicity islet. Infect Immun. 2006;74(4):2453-2456.

39. Nelson AL, et al. RrgA is a pilus-associated adhesin in Streptococcus pneumoniae. Mol Microbiol. 2007;66(2):329-340.

40. El Mortaji L, Fenel D, Vernet T, Di Guilmi AM. Association of RrgA and RrgC into the Streptococcus pneumoniae pilus by sortases $\mathrm{C}-2$ and C-3. Biochemistry. 2012;51(1):342-352.

41. Stryker LM. Variations in the pneumococcus induced by growth in immune serum. J Exp Med. 1916;24(1):49-68.

42. Jacquot J, Hayem A, Galabert C. Functions of proteins and lipids in airway secretions. Eur Respir J. 1992;5(3):343-358.

43. Apter FM, Lencer WI, Finkelstein RA, Mekalanos JJ, Neutra MR. Monoclonal immunoglobulin A antibodies directed against cholera toxin prevent the toxin-induced chloride secretory response and block toxin binding to intestinal epithelial cells in vitro. Infect Immun. 1993;61(12):5271-5278.

44. Stubbe H, Berdoz J, Kraehenbuhl JP, Corthésy B. Polymeric IgA is superior to monomeric IgA and IgG carrying the same variable domain in preventing Clostridium difficile toxin A damaging of T84 monolayers. JImmunol. 2000;164(4):1952-1960.

45. Kauppi-Korkeila M, van Alphen L, Madore D, Saarinen L, Käyhty H. Mechanism of antibody-mediated reduction of nasopharyngeal colonization by Haemophilus influenzae type $\mathrm{b}$ studied in an infant rat model. J Infect Dis. 1996;174(6):1337-1340.

46. Forbes SJ, Eschmann M, Mantis NJ. Inhibition of Salmonella enterica serovar typhimurium motility and entry into epithelial cells by a protective antilipopolysaccharide monoclonal immunoglobulin A antibody. Infect Immun . 2008;76(9):4137-4144.

47. Dallas SD, Rolfe RD. Binding of Clostridium difficile toxin A to human milk secretory component. JMed Microbiol. 1998;47(10):879-888.

48. Bender MH, Weiser JN. The atypical aminosterminal LPNTG-containing domain of the pneumococcal human IgA1-specific protease is required for proper enzyme localization and function. Mol Microbiol. 2006;61(2):526-543.

49. Janoff EN, et al. Pneumococcal IgA1 protease subverts specific protection by human IgA1. Mucosal Immunol. 2014;7(2):249-256.

50. Kilian M, Thomsen B, Petersen TE, Bleeg HS. Occurrence and nature of bacterial IgA proteases. Ann N Y Acad Sci. 1983;409:612-624.

51. Wani JH, Gilbert JV, Plaut AG, Weiser JN. Identification, cloning, and sequencing of the immunoglobulin A1 protease gene of Streptococcus pneumoniae. Infect Immun. 1996;64(10):3967-3974.

52. Weiser JN, Bae D, Fasching C, Scamurra RW,
Ratner AJ, Janoff EN. Antibody-enhanced pneumococcal adherence requires IgA1 protease. Proc Natl Acad Sci U S A. 2003;100(7):4215.

53. Chewapreecha $C$, et al. Dense genomic sampling identifies highways of pneumococcal recombination. Nat Genet. 2014;46(3):305-309.

54 . Turner P, et al. Serum antibody responses to pneumococcal colonization in the first 2 years of life: results from an SE Asian longitudinal cohort study. Clin Microbiol Infect. 2013;19(12):E551-E558.

55. Corander J, et al. Frequency-dependent selection in vaccine-associated pneumococcal population dynamics. Nat Ecol Evol. 2017;1(12):1950-1960.

56. Roche AM, Richard AL, Rahkola JT, Janoff EN, Weiser JN. Antibody blocks acquisition of bacterial colonization through agglutination. Mucosal Immunol. 2015;8(1):176-185.

57. Fasching CE, Grossman T, Corthésy B, Plaut AG, Weiser JN, Janoff EN. Impact of the molecular form of immunoglobulin A on functional activity in defense against Streptococcus pneumoniae. Infect Immun. 2007;75(4):1801-1810.

58. Phalipon A, Cardona A, Kraehenbuhl JP, Edelman L, Sansonetti PJ, Corthésy B. Secretory component: a new role in secretory IgA-mediated immune exclusion in vivo. Immunity. 2002;17(1):107-115.

59. Alvarez N, et al. Passive administration of purified secretory IgA from human colostrum induces protection against Mycobacterium tuberculosis in a murine model of progressive pulmonary infection. BMC Immunol. 2013;14(suppl 1):S3.

60. Bioley G, Monnerat J, Lötscher M, Vonarburg C, Zuercher A, Corthésy B. Plasma-derived polyreactive secretory-like IgA and IgM opsonizing Sal monella enterica typhimurium reduces invasion and gut tissue inflammation through agglutination. Front Immunol. 2017;8:1043.

61. Mitsi E, et al. Agglutination by anti-capsular polysaccharide antibody is associated with protection against experimental human pneumococcal carriage. Mucosal Immunol. 2017;10(2):385-394.

62. Clamp JR. The relationship between secretory immunoglobulin A and mucus [proceedings]. Biochem Soc Trans. 1977;5(5):1579-1581.

63. Gleeson M, Cripps AW. Development of mucosal immunity in the first year of life and relationship to sudden infant death syndrome. FEMS Immunol Med Microbiol. 2004;42(1):21-33.

64. Borges IC, et al. Natural development of antibodies against Streptococcus pneumoniae, Haemophilus influenzae, and Moraxella catarrhalis protein antigens during the first 13 years of life. Clin Vaccine Immunol. 2016;23(11):878-883.

65. Croucher NJ, et al. Population genomics of post-vaccine changes in pneumococcal epidemiology. Nat Genet. 2013;45(6):656-663.

66. Li Y, Thompson CM, Lipsitch M. A modified Janus cassette (Sweet Janus) to improve allelic replacement efficiency by high-stringency negative selection in Streptococcus pneumoniae. PLoS One. 2014;9(6):e100510.

67. Mukerji R, et al. Pneumococcal surface protein A inhibits complement deposition on the pneumococcal surface by competing with the binding of C-reactive protein to cell-surface phosphocholine. JImmunol. 2012;189(11):5327-5335.

68. Kono M, Zafar MA, Zuniga M, Roche AM, Hamaguchi S, Weiser JN. Single cell bottlenecks in the pathogenesis of Streptococcus pneumoniae. PLoS Pathog. 2016;12(10):e1005887.

69. Shuter J, Hatcher VB, Lowy FD. Staphylococcus aureus binding to human nasal mucin. Infect Immun. 1996;64(1):310-318.

70. Fukuda M. Chemical labeling of carbohydrates by oxidation and sodium borohydride reduction. Curr Protoc Mol Biol. 2001; Chapter 17:Unit 17.5.

71. Guthrie RD. The "Dialdehydes" from the periodate oxidation of carbohydrates. In: Wolfrom ML, ed. Advances in Carbohydrate Chemistry. Boston, Massachusetts, USA; Academic Press; 1962:105-158.

72. Prakash R, Bharathi Raja S, Devaraj H, Devaraj SN. Up-regulation of MUC2 and IL-1 $\beta$ expression in human colonic epithelial cells by Shigella and its interaction with mucins. PLoS One. 2011;6(11):e27046.

73. Basset A, Turner KH, Boush E, Sayeed S, Dove SL, Malley R. Expression of the type 1 pneumococcal pilus is bistable and negatively regulated by the structural component RrgA. Infect Immun. 2011;79(8):2974-2983.

74. Zhang Z, Clarke TB, Weiser JN. Cellular effectors mediating Th17-dependent clearance of pneumococcal colonization in mice. JClin Invest. 2009;119(7):1899-1909.

75. Turner P, et al. A longitudinal study of Streptococcus pneumoniae carriage in a cohort of infants and their mothers on the ThailandMyanmar border. PLoS One. 2012;7(5):e38271.

76. Lees JA, et al. Genome-wide identification of lineage and locus specific variation associated with pneumococcal carriage duration. Elife. 2017;6:e26255.

77. Lees JA, et al. Sequence element enrichment analysis to determine the genetic basis of bacterial phenotypes. Nat Commun. 2016;7:12797.

78. Earle SG, et al. Identifying lineage effects when controlling for population structure improves power in bacterial association studies. Nat Microbiol. 2016;1:16041.

79. Lees JA, Galardini M, Bentley SD, Weiser JN, Corander J. pyseer: a comprehensive tool for microbial pangenome-wide association studies. Bioinformatics. 2018;34(24):4310-4312.

80. Zafar MA, Kono M, Wang Y, Zangari T, Weiser JN. Infant mouse model for the study of shedding and transmission during Streptococcus pneumoniae monoinfection. Infect Immun. 2016;84(9):2714-2722.

81. Shen P, Lees JA, Bee GCW, Brown SP, Weiser JN. Pneumococcal quorum sensing drives an asymmetric owner-intruder competitive strategy during carriage via the competence regulon. Nat Microbiol. 2019;4(1):198-208. 